\title{
Transfer Learning for Tilt-Dependent Radio Map Prediction
}

\author{
Claudia Parera ${ }^{\left({ }^{\circ}\right.}$, Qi Liao $^{\left({ }^{(}\right)}$, Ilaria Malanchini $\left.{ }^{(}\right)$, Cristian Tatino ${ }^{(0)}$, \\ Alessandro E. C. Redondi ${ }^{(}$, and Matteo Cesana ${ }^{(i)}$
}

\begin{abstract}
Machine learning will play a major role in handling 2 the complexity of future mobile wireless networks by improving 3 network management and orchestration capabilities. Due to the 4 large number of parameters that can be monitored and config5 ured in the network, collecting and processing high volumes of 6 data is often unfeasible or too expensive at network runtime, 7 which calls for taking resource management and service orches8 tration decisions with only a partial view of the network status. 9 Motivated by this fact, this paper proposes a transfer learning 10 framework for reconstructing the radio map corresponding to a 11 target antenna tilt configuration by transferring the knowledge 12 acquired from another tilt configuration of the same antenna, 13 when no or very limited measurements are available from the 14 target. The performance of the framework is validated against 15 standard machine learning techniques on a data set collected 16 from a 4G commercial base stations. In most of the tested scenar17 ios, the proposed framework achieves notable prediction accuracy 18 with respect to classical machine learning approaches, with a 19 mean absolute percentage error below $8 \%$.
\end{abstract}

Index Terms-Radio map prediction, antenna tilt, transfer 21 learning.

22

${ }_{23}$ IFTH generation wireless networks (5G) are expected to ${ }_{24} 1$ improve the performance of cellular systems, achieving 25 higher data rates, reduced latency, higher reliability and sup${ }_{26}$ port for greater numbers of users. To achieve this, $5 \mathrm{G}$ resorts 27 to dense and heterogeneous deployments, coupled with higher ${ }_{28}$ flexibility in the network access and core domains, which can 29 be dynamically managed in either a centralized or distributed so manner. To cope with such a complex scenario, it is foreseen ${ }_{31}$ that machine learning tools will play a major role in enabling

Manuscript received June 25, 2019; revised October 31, 2019; accepted December 27, 2019. This work was supported by the European Union's Horizon 2020 Research and Innovation Programme under the Marie Sklodowska-Curie Grant Agreement 643002. This work extends the preliminary study in [1]. The associate editor coordinating the review of this article and approving it for publication was W. Saad. (Corresponding author: Claudia Parera.)

Claudia Parera is with DEIB, Politecnico di Milano, 20133 Milan, Italy, and also with Bell Labs, Nokia, 70435 Stuttgart, Germany (e-mail: claudia. parera@polimi.it).

Qi Liao and Ilaria Malanchini are with Bell Labs, Nokia, 70435 Stuttgart, Germany (e-mail: qi.liao@nokia-bell-labs.com; ilaria.malanchini@ nokia-bell-labs.com).

Cristian Tatino is with ITN, Linköping University, 60221 Linköping, Sweden (e-mail: cristian.tatino@liu.se).

Alessandro E. C. Redondi and Matteo Cesana are with DEIB, Politecnico di Milano, 20133 Milan, Italy (e-mail: alessandroenrico.redondi@ polimi.it; matteo.cesana@polimi.it).

Digital Object Identifier 10.1109/TCCN.2020.2964761 the transition from current mobile networks to future 5G archi- 32 tectures [2]. By exploiting the increased availability of data in $5 \mathrm{G}$ coming from network devices and user terminals, machine learning tools will be able to assist network operators in dealing with the increasing complexity of configuring parameters for network optimization. Thus, machine learning tools will form the basis for automated and smart network management techniques.

Among the manifold parameters that can be configured at 40 the base station (BS), one of the most important is the antenna ${ }_{41}$ tilt, which is the angle formed by the vertical direction which 42 the antenna is facing and the horizon. Antenna tilt can be con- 43 trolled either mechanically (by physically tilting the antenna ${ }_{44}$ up or down) or electronically (relying on beam-forming tech- 45 niques that steer the main beam of the antenna towards a ${ }_{46}$ desired vertical direction), or by a combination of the two. ${ }_{47}$ The antenna tilt directly impacts the performance of the cell ${ }_{48}$ served by the BS in terms of network coverage, signal strength 49 and inter-cell interference, and therefore determines the qual- 50 ity of service experienced by end users. In particular, when the 51 antenna tilt is changed in the BS, its effect on the antenna gain 52 over distance also changes, which further leads to a change ${ }_{53}$ of the Reference Signals Received Power (RSRP) values [3]. 54 Therefore, different radio maps can be generated as a func- 55 tion of the selected tilt configuration. We refer to these as 56 tilt-dependent radio maps.

From an operator's perspective being able to predict cell ${ }_{58}$ performance without carrying out extensive trials or mea- 59 surement campaigns is of key importance for two reasons: 60 firstly, extensive measurement campaigns, such as test driving, 61 are time consuming and costly. Secondly, even if measure- 62 ments were obtained inexpensively (e.g., directly from user 63 terminals through crowd-sourcing), testing all possible antenna 64 configurations might still be impractical at network runtime. ${ }_{65}$

Given such difficulties, a solution which is particularly 66 appealing to network operators is transferring the knowl- ${ }_{67}$ edge acquired from a single measurement campaign (for a 68 given antenna tilt setting) to a new domain (a new tilt set- 69 ting) without needing to acquire a complete set of additional 70 measurements. In this case, the data distributions of the train- 71 ing (source) and testing (target) sets are different. Therefore, 72 we formalize and solve this problem via transfer learning, a 73 paradigm that has received increasing attention in the last few 74 years [4].

In this paper, we study the possibility of performing transfer 76 learning for the task of predicting the radio signal strength 77 
78 map of a particular BS. We start from a dataset of sig79 nal strength measurements collected from commercial, Long 80 Term Evolution (LTE) BSs and analyze the performance of a 81 transfer learning approach based on a deep neural network, 82 where a domain is defined as the knowledge acquired for 83 a particular antenna tilt setting. This is then transferred to 84 a different domain, i.e., a different tilt configuration of the ${ }_{85}$ same antenna. As a benchmark, we compare the performance 86 of our proposed method against the performance of stan87 dard machine learning techniques when applied to the same 88 problem. The performance evaluation is carried out in two 89 different scenarios: firstly, we use a single tilt configura90 tion as the source domain. Secondly, we augment the source 91 domain by adding data available from other tilt configura92 tions of the same antenna. We study the behavior of the 93 proposed transfer learning approach when the data available 94 from the target tilt configuration is limited, and further ana${ }_{95}$ lyze different strategies to select the limited points of the target 96 domain.

In summary, the main contributions of this paper are as 98 follows:

- We propose a transfer learning framework based on deep neural networks, i.e., Feed-Forward Neural networks (FFNs), for tilt-dependent radio map prediction. Contrary to work in the area of deep and transfer learning for computer vision and natural language processing, where more complex architectures (Convolutional Neural Networks (CNNs), BERT [5] and ULMFit [6]) are used, we evaluate the joint use of a simpler FFN architecture and transfer learning for a different kind of data, namely, radio access network data.

- We describe the optimization of the reference architecture, as well as of the parameters for both source and target domains. The system is trained exclusively on data coming from an existing network deployment.

- Through numerical experiments, we evaluate prediction performance against standard machine learning approaches. The proposed approach is shown to achieve notable prediction accuracy, specifically when the amount of data available from the target domain is limited. Moreover, under realistic assumptions on the data availability, we show the scenarios where transfer learning leads to performance improvements. Finally, we show how data augmentation leads to further performance improvement.

The rest of this paper is organized as follows: Section II 124 reviews related works in the area of radio map prediction, 125 with a particular focus on those works dealing with antenna 126 tilt. It further reviews the state of the art of transfer learn127 ing and its applications in the area of network planning and 128 optimization. Section III describes in detail the scenario out129 lined above, as well as preliminary data collection and data 130 pre-processing steps. Section IV focuses on the machine learn${ }_{131}$ ing tools used for this work. Experiments and discussion of 132 the obtained results, with special emphasis on the use cases 133 where transfer learning outperforms traditional machine learn134 ing approaches, are reported in Section V. Finally, Section VI 135 concludes the paper.

\section{BACKGROUND AND RELATED WORK}

In this section, we briefly review the works on antenna 137 tilt-dependent radio map prediction, introduce background 138 information on transfer learning, and comment on related 139 applications of transfer learning techniques to wireless 140 networks.

\section{A. Tilt-Dependent Radio Map Prediction}

Tilt-dependent radio map prediction plays a crucial role ${ }_{143}$ in the context of network planning and proactive network 144 optimization [7]. The predicted propagation condition can be 145 exploited for a reliable decision making process to dynami- ${ }_{146}$ cally optimize antenna tilts in a time-varying network envi- 147 ronment [7], [8]. Although radio map prediction has been 148 extensively studied [9], its dependency on antenna tilt has been 149 investigated only in few works. The authors in [10] propose a 150 geometrical-based extension to various traditional log-distance 151 path loss models (Okumura-Hata, Walfisch-Ikegami) to take 152 into account the antenna tilt during the prediction of the sig- 153 nal strength at a given distance from the BS. The proposed 154 extension, named vertical gain correction (VGC), is calculated 155 directly from the antenna patterns provided by the manufac- 156 turer and is added to the signal strength estimated by the path 157 loss models to compensate for the antenna tilt. Experimental 158 results on data collected from LTE BSs show that VGC ${ }_{159}$ improves the performance of signal strength prediction when 160 compared to traditional models. Similarly, the work in [11] 161 investigates the effect of antenna tilt on radio maps, com- 162 paring the path loss models developed by the 3rd generation 163 partnership project (3GPP) [12] for different propagation envi- 164 ronments. The results were obtained using a ray tracing tool 165 able to take into account antenna tilts and demonstrate that 166 changing antenna tilt has a significant impact on the shad- 167 owing map. This calls for a rethinking of currently available 168 3GPP propagation models and assumptions, which apply an 169 identical shadowing map independently from the antenna tilt. 170

\section{B. Overview of Transfer Learning}

171

Traditional machine learning algorithms work under the 172 assumptions that training and testing data are taken from the 173 same distribution and have the same feature space. However, 174 in real world applications these assumptions do not always 175 hold. Firstly, the data distribution may not be static, but vary 176 over time, making it difficult to apply a trained model to a 177 new scenario at a different time period. Secondly, training and 178 testing data could also differ in terms of geographic location, 179 or the equipment used for recording the measurements (e.g., 180 a different mobile device). In such cases, transfer learning is 181 a promising approach for exploiting and sharing knowledge 182 among different domains.

183

In this paper, a domain $\mathcal{D}:=\{\mathcal{X}, P(X)\}$ consists of a fea- ${ }_{184}$ ture space $\mathcal{X}$ and its probability distribution $P(X), X \in \mathcal{X} .{ }_{185}$ A task $\mathcal{T}:=\{\mathcal{Y}, f(\cdot)\}$ consists of a label space $\mathcal{Y}$ and a 186 predictive function $f(\cdot)$, where $f(\cdot)$ can be written as $P(Y \mid X), 187$ $Y \in \mathcal{Y}$ and $X \in \mathcal{X}$. Formally, the definition of transfer 188 learning is given as follows. 
190 Definition 1 (Transfer Learning [4]): Given a source 191 domain $\mathcal{D}_{S}$ and learning task $\mathcal{T}_{S}$, a target domain $\mathcal{D}_{T}$ and 192 learning task $\mathcal{T}_{T}$, transfer learning aims to improve the learn193 ing of the target predictive function $f_{T}(\cdot)$ in $\mathcal{D}_{T}$ using the 194 knowledge in $\mathcal{D}_{S}$ and $\mathcal{T}_{S}$, where $\mathcal{D}_{S} \neq \mathcal{D}_{T}$, or $\mathcal{T}_{S} \neq \mathcal{T}_{T}$.

195 Three seminal papers [4], [13], [14] review the state of the 196 art of transfer learning in classification, regression, unsuper197 vised and reinforcement learning. When dealing with a transfer 198 learning problem, the main research questions are: what to 199 transfer, how to transfer and when to transfer. When to trans$200 \mathrm{fer}$ is mainly related to the issue of avoiding negative transfer, 201 which happens when transfer learning has a negative impact 202 on the performance of target learning. The literature is pri203 marily focused on the first two questions. For the purpose of 204 studying what to transfer, we will use the categorization found 205 in [4], where transfer learning can be divided into:

206 - Inductive transfer: Different source and target tasks and 207 same or different source and target domains.

208 - Transductive transfer: Same source and target tasks but different source and target domains.

- Unsupervised transfer: Similar to inductive transfer, with different but related source and target tasks. The focus is solving an unsupervised learning task on the target domain. There is no labeled data available from source and target domains during training.

To answer the question how to transfer, the most common 16 transfer learning approaches are:

- Instance transfer: Labeled samples in the source domain are reweighted and used in the target domain; it can be applied to inductive and transductive learning [15], [16], [17].

- Feature transfer: Aims at finding a 'good' feature representation that can minimize the domain difference, as proposed in [18], [19]; it is applied to inductive and transductive learning.

- Parameter transfer: Works under the assumption that individual models for related tasks share parameters or a combination of hyperparameters [20], [21]; it is mostly applied to inductive and transductive transfer learning.

- Relational knowledge transfer: Is applied to problems where there is some kind of relation in the data (e.g., network or social network data) [22], [23]; it is mostly applied to inductive transfer learning.

\section{${ }_{233}$ C. Applications of Transfer Learning}

234 Some of the areas where transfer learning has been success235 fully applied are computer vision, natural language processing 236 and speech recognition [4]. Due to recent advances in the field 237 of deep learning, recent approaches combine deep and transfer 238 learning. For instance, in [24] mid-level image representations 239 learned with a $\mathrm{CNN}$ are transferred to other visual recogni240 tion tasks. The same idea is followed in [25] for character 241 recognition from Latin to Chinese. However, the applica242 tions of transfer learning in the field of wireless and mobile 243 networks are still limited [26]. Work has been done for local244 ization by transferring knowledge across devices, time and 245 space in [27], [28], [29], [30]. More recently, transfer learning has been applied to caching [31], resources optimization [32], 246 fault classification [33] and resource management in Wireless 247 Virtual Reality [34].

\section{Motivation of Our Study}

Our work shares the same research objectives as the work 250 on tilt-dependent radio map prediction (Section II-A), but with 251 one fundamental difference: in all the aforementioned works 252 the source domain for predicting the signal strength is similar 253 to, or the same as, the target domain. For example, the signal 254 strength radio map of an antenna under a given tilting con- 255 figuration is predicted using available signal strength samples 256 collected for the same antenna in the same tilt configuration. 257 Instead, we analyze the case where the performance of the 258 target antenna configuration is predicted using training data 259 from a different tilt configuration. In our previous work [1], 260 we investigated the dependency between the transferability of 261 the knowledge and the domain difference, when considering 262 the task of tilt-dependent radio map prediction and by using 263 standard machine learning tools. In this work, we aim to solve 264 a similar problem, with improved performance, by applying 265 transfer learning and exploiting different data sources as source 266 and target domains.

Our work mainly falls into the category of transductive 268 learning $\left(\mathcal{T}_{S}=\mathcal{T}_{T}\right.$ and $\left.\mathcal{D}_{S} \neq \mathcal{D}_{T}\right)$. Furthermore, our solu- 269 tion is inspired by the feature transfer and parameter transfer 270 approaches introduced in [24], where the authors propose to 271 extract some internal layers from a CNN, trained with suf- 272 ficient data collected from the source domain. They add an 273 adaptation layer to correct the difference between distribu- 274 tions in the source and target domain. The resulting network is 275 trained with a limited amount of data from the target domain. 276 However, unlike the approach proposed in [24], we do not 277 use CNNs, due to the format, scarcity and small feature space 278 dimension (i.e., geospatial information and Reference Signal 279 Received Power (RSRP) values) of the collected data. For the 280 same reasons, we do not consider recurrent architectures such 281 as Long Short-Term Memory (LSTM) networks, which have 282 been adopted successfully for problems where data, unlike 283 the current radio maps, has a strong temporal structure (e.g., 284 time series prediction or speech recognition) [35]. Instead, we 285 take the internal layers of a fully connected FFN, trained in a 286 given tilt configuration (source domain), and add a new layer. 287 Then, we retrain the final network on a new tilt configura- 288 tion (target domain). To exploit parameter transfer, we assume 289 that different domains share the same combination of hyper- 290 parameters (same neural network architecture). In addition, 291 we quantify the minimum amount of labeled data required 292 from the target domain to carry out predictions. We showcase 293 real world scenarios where a transfer learning solution out- 294 performs traditional machine learning algorithms. Finally, we 295 show how augmenting the source domain by adding data from 296 other available tilt configurations of the same antenna helps to 297 improve the performance of our transfer learning approach. To 298 the best of our knowledge, this is the first work transferring 299 knowledge across different network configurations by partially 300 
${ }_{301}$ retraining deep neural networks, in the area of wireless and 302 mobile networks.

\section{Problem Statement and Dataset}

304 We address the following problem: "how to predict the 305 performance of a given network configuration by leverag306 ing information from different network configurations". The 307 performance measure that we target is the received signal 308 strength in the downlink. The network configuration domains 309 include the tilting configurations of the transmitting BSs.

${ }_{310}$ We consider a BS that can work in $H$ different tilt configu311 rations, indexed by $h=1, \ldots, H$. Let $s_{h}\left(\mathbf{x}_{i}\right)$ be the measured 312 signal strength received at location $\mathbf{x}_{i}=\left\{y_{i}, z_{i}\right\}$ when the $h$-th 313 tilt configuration is selected at the BS, where $y_{i}$ and $z_{i}$ indicate 314 the latitude and the longitude of the $i$-th location, respectively. ${ }_{315}$ Let $\mathcal{M}_{h}$ be the set of location indexes where measurements 316 have been taken with configuration $h$.

317 The problem at hand can be defined as follows: given ${ }_{318}\left\{s_{h}\left(\mathbf{x}_{i}\right): i \in \mathcal{M}_{h}\right\}$, estimate the unknown signal strength ${ }_{319} \hat{s}_{n}\left(\mathbf{x}_{j}\right)$ at the same or different locations, $\mathbf{x}_{j}$, with $j \in \mathcal{M}_{n}$, 320 under different network configuration domains, $n \neq h$.

\section{A. Data Collection}

322 The dataset used in this work is composed of RSRP out323 door measurements collected in Espoo, Finland, in November 3242016 from two commercial LTE BSs with three different $120^{\circ}$ 325 sectors each and operating at $2.6 \mathrm{GHz}$. Figure 1 shows the 326 positions of the two antennas and the representation of the 327 target area. The measurements were collected from three dif328 ferent Physical Cell Identifiers (PCIs), which will be referred 329 to as PCI 1,2 and 3. PCIs 1 and 2 refer to two different 330 sectors of the same BSs, whereas PCI 3 is a sector of a dif331 ferent BS. The RSRP measurements were collected using an 332 Android device equipped with an application capable of stor${ }_{3 з 3}$ ing the RSRP from all the received cells, the cell identifier, 334 the Global Positioning System (GPS) position of the device 335 and the timestamp. Such measurements were carried out at 336 a frequency of $1 \mathrm{~Hz}$ while walking along routes of $8 \mathrm{~km}$ 337 within each cell coverage area, with a minimum and maxi338 mum distance from the BS of $30 \mathrm{~m}$ and $900 \mathrm{~m}$, respectively. 3з9 By design, the testing paths were planned to include different 340 propagation conditions: university campus with two or three341 story buildings, residential areas, parking lots, lower density 342 rural and open areas with different types of roads (e.g., pedes343 trian, cycling and main roads). Each testing path was walked 344 once for each electronic tilt setting. The available tilt settings 345 are 2, 3 and 6 degrees for each PCI, respectively. The receiver 346 was placed at the height of $1.5 \mathrm{~m}$ and always kept at the same 347 orientation. The weather conditions were stable and cloudy, 348 and the route was covered by snow for most of the mea349 surement campaign. The RSRP values were collected from 350 an operating mobile network. According to [36], these values 351 include the power from co-channel serving and non-serving 352 cells as well as adjacent channel interference, but only on 353 the resource elements that carry reference signals. Since these 354 values are measured only in the symbols carrying the refer355 ence signal, they exclude most of the wide band noise and

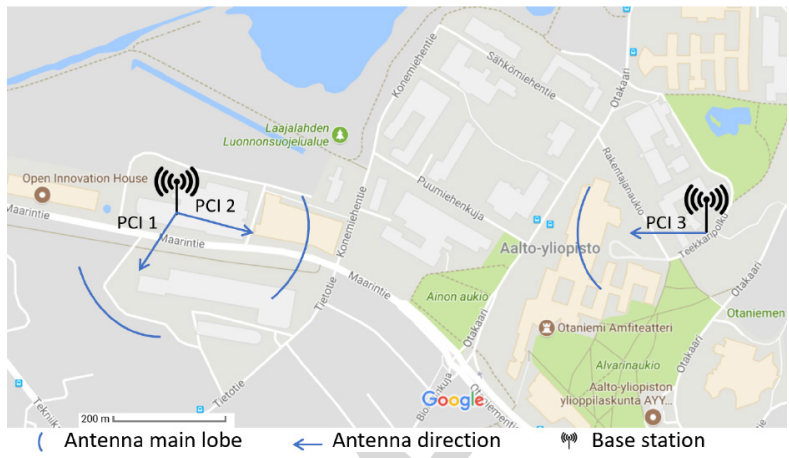

Fig. 1. Map showing the BS positions and the PCIs in the reference dataset.

interference from other cells. Overall, they are proportional ${ }_{356}$ to the SNR on average [37]. Therefore, they are still a good ${ }_{357}$ indicator to be used in radio map reconstruction, reflecting the 358 channel propagation conditions.

\section{B. Data Preprocessing}

360

In total, about $3 \cdot 10^{5}$ RSRP measurements were obtained. 361 Each observation contains the following fields: 362

- Measurement position (latitude and longitude coordi- 363 nates)

- RSRP value (downlink signal strength)

- PCI (physical cell identifier)

The raw dataset was preprocessed to remove corrupted sam- 367 ples: for example, at the beginning of each experiment the ${ }_{368}$ GPS receiver requires some initialization time during which 369 position is recorded incorrectly. Moreover, we overlaid the 370 considered area with a grid. For each grid element of size 371 $20 \mathrm{~m} \times 20 \mathrm{~m}$, we replaced the RSRP values with their aver- 372 age to reduce noise. After the preprocessing steps, the reduced 373 dataset consisted of $\sim 600$ observations per PCI and per tilt ${ }_{374}$ configuration, for a total of $\sim 5.8 \cdot 10^{3}$ measurements. Before 375 training, the data is scaled between 0 and 1 by using a Min- 376 Max scaler, which is fitted to the training set and applied to 377 the cross validation and test sets. The scaling transformation is 378 then reversed before evaluating the algorithm performance. In 379 our previous work [1], we analyze the transferability across 380 different tilt settings of the same PCI as well as the trans- ${ }_{381}$ ferability across different PCIs. In particular, we show that 382 the transferability within the same PCI is much higher than 383 the transferability across different PCIs. Therefore, we focus 384 hereafter on the task of transferring the knowledge from one 385 tilt configuration to another within the same PCI. Unlike our 386 previous work, in which the standard machine learning tools 387 are used, we apply transfer learning with deep neural networks. 388

Figure 2 shows a representation of the data collected for 389 different tilt configurations of PCI 1. Figures 2(a), 2(c), 2(e) 390 show RSRP values (in $\mathrm{dBm}$ ) over the considered geographic 391 area, when the antenna was tilted at 2, 3 and 6 degrees, 392 respectively. It can be observed that the spatial distribution ${ }_{393}$ of the data follows a similar pattern for different tilt config- 394 urations of PCI 1. For example, points located in the main 395 direction of the antenna have higher signal strength values 396 than the rest of the points. In addition, points closer to the 397 


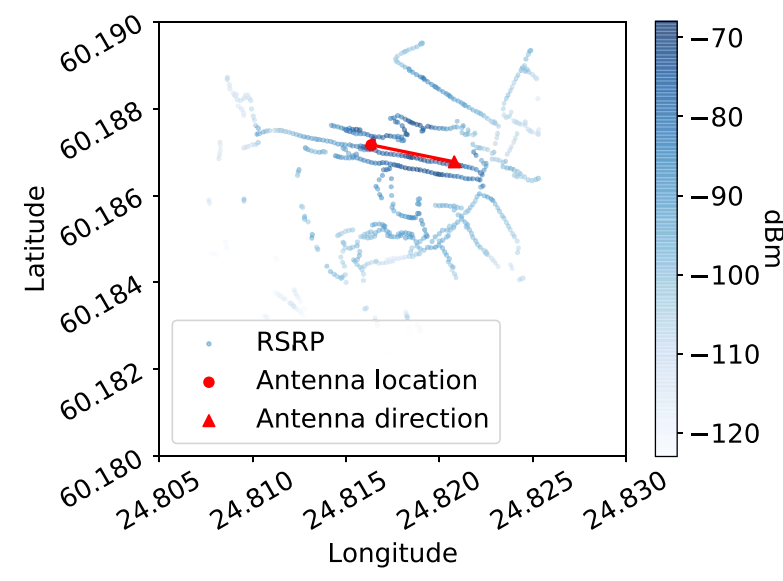

(a) RSRP values at the sampled points, Tilt 2

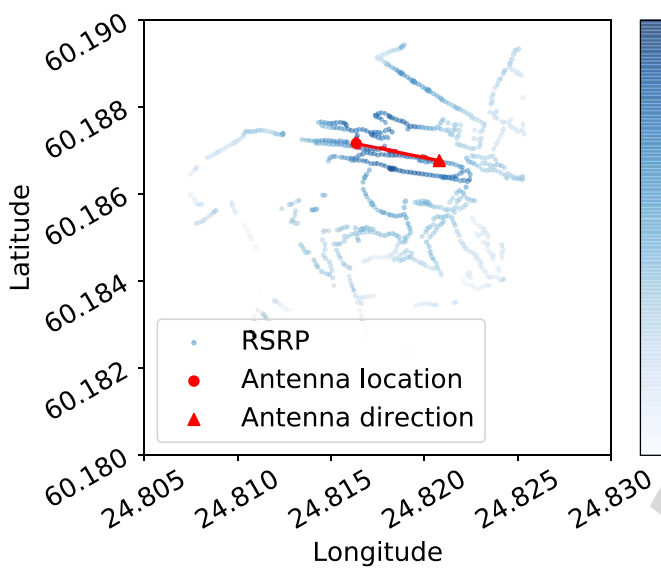

(c) RSRP values at the sampled points, Tilt 3

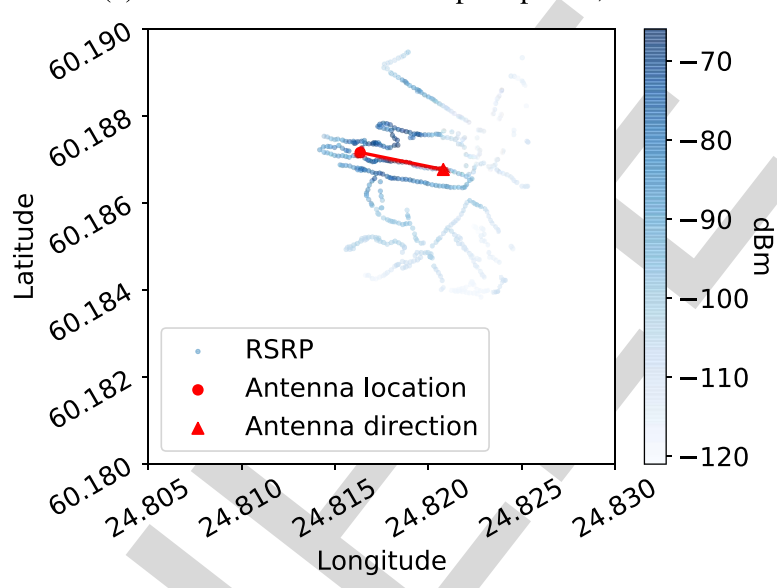

(e) RSRP values at the sampled points, Tilt 6

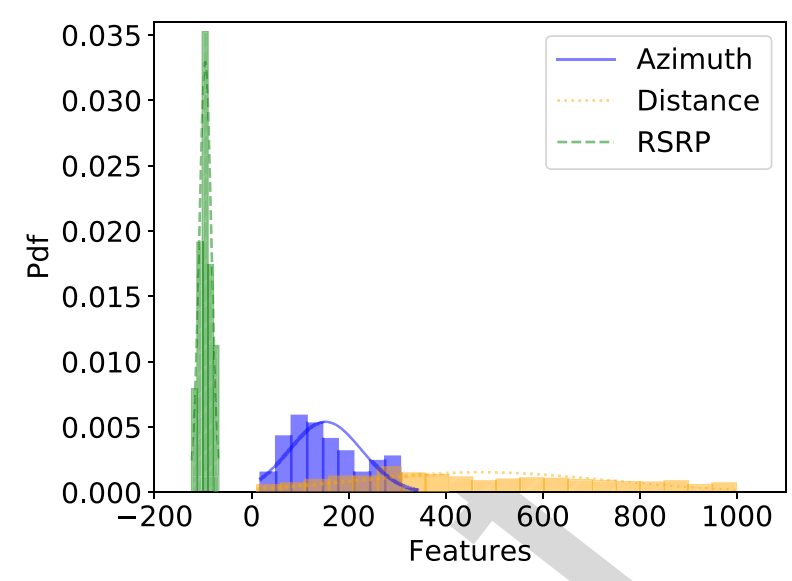

(b) Probability Density Function (PDF), Tilt 2

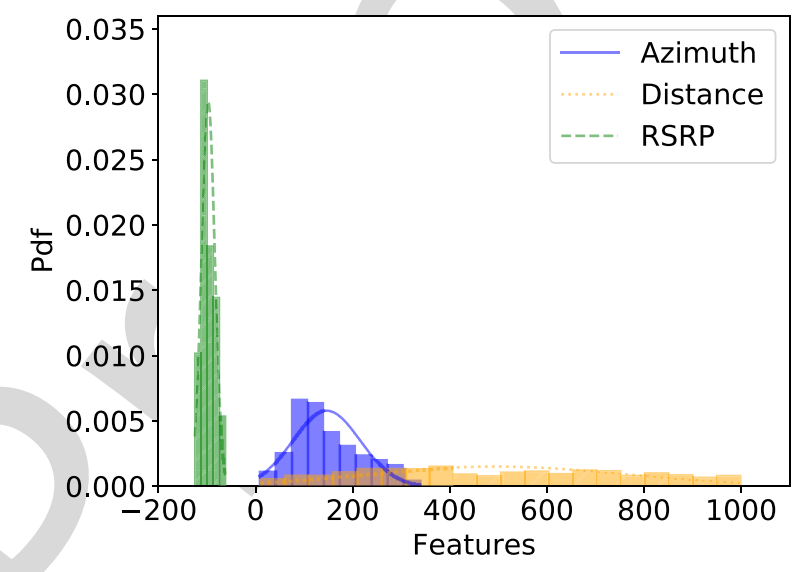

(d) PDF, Tilt 3

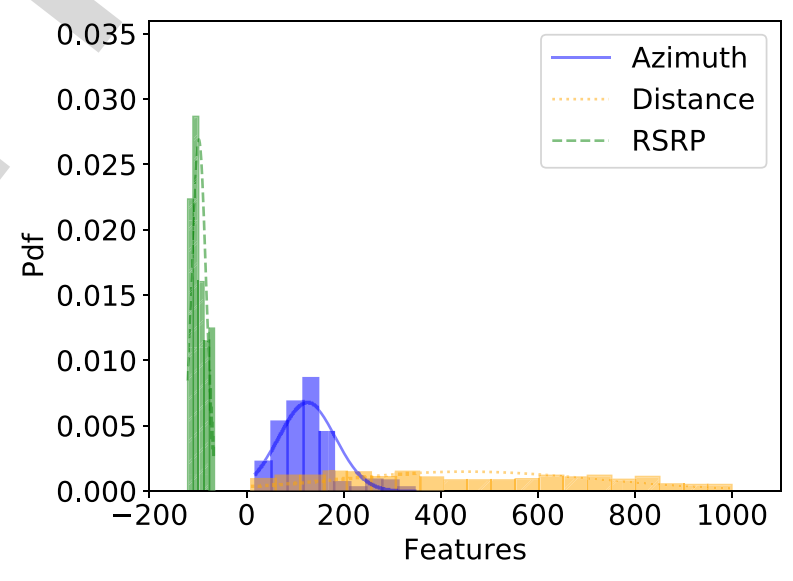

(f) PDF, Tilt 6

Fig. 2. Tilt-dependent radio maps, normalized histograms and Probability Density Functions (PDFs) for three metrics RSRP, azimuth and distance, PCI 1.

398 antenna also follow a similar pattern, while points which are 399 far apart have lower RSRP values. To give an idea of the 400 domain differences, in Figures 2(b), 2(d), 2(f) we show the 401 normalized histograms, and the continuous approximations of 402 the PDFs of the azimuth, distance and RSRP for the different 403 tilt configurations of PCI 1.

404 Even if some similarities can be observed between the sta405 tistical characteristics of the data collected under different 406 tilt configurations, the data does not come from the same distributions. For example, the azimuth distribution for a 407 greater tilt value (Figure 2(f)) has a lower standard devia- 408 tion than the distributions for lower tilt values (Figures 2(b) 409 and 2(d)).

\section{Prediction Approaches}

Given the base station location $\mathbf{x}_{A}$, let $\mathbf{x}$ and $h$ be the tar- ${ }_{412}$ get position and the configured antenna tilt, respectively. The ${ }_{413}$ 


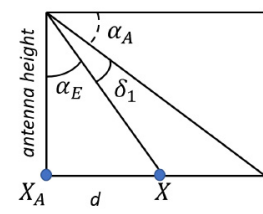

(a) Relative distance

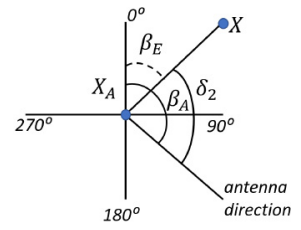

(b) Relative azimuth
Fig. 3. Relative angles on the vertical (left) and horizontal (right) planes between the antenna pointing direction and the direction towards the test position $\mathbf{x}$.

${ }_{414}$ following set of features, derived from $(\mathbf{x}, h)$ and shown in 415 Figure 3, is considered for the prediction task:

${ }_{416}$ - the physical distance between the antenna and the measurement position, $d(\mathbf{x}):=d\left(\mathbf{x}, \mathbf{x}_{A}\right)$

- the relative elevation angle between the down-tilt of the antenna and the vertical direction from the antenna emitting element to the measurement position, defined as:

$$
\begin{aligned}
\delta_{1}(h, \mathbf{x}) & =90^{\circ}-\left(\alpha_{A}+\alpha_{E}\left(\mathbf{x}, \mathbf{x}_{A}\right)\right) \\
& =90^{\circ}-\left(h+\alpha_{E}\left(\mathbf{x}, \mathbf{x}_{A}\right)\right),
\end{aligned}
$$

where $\alpha_{A}:=h$ is the antenna down-tilt (mechanical plus electrical) and $\alpha_{E}$ is the angle at which the antenna 'sees' the target position depending on the antenna position $\mathbf{x}_{A}$ and the target location $\mathbf{x}$

- the relative azimuth between the horizontal orientation of the antenna and the horizontal direction to the measurement position defined as:

$$
\delta_{2}(\mathbf{x})=\beta_{A}-\beta_{E}\left(\mathbf{x}, \mathbf{x}_{A}\right),
$$

where $\beta_{A}$ denotes the horizontal orientation of the antenna and $\beta_{E}$ is the horizontal orientation of the target position with respect to the antenna position

Each sample in the training dataset is, therefore, associated 435 with a tuple of values $\left(d, \delta_{1}, \delta_{2}\right)$. The logarithmic transfor${ }_{436}$ mation is applied to $d$ since the RSRP values are measured ${ }_{437}$ in $\mathrm{dBm}$. Finally the feature vector $\left[d(\mathbf{x}), \delta_{1}(\mathbf{x}, h), \delta_{2}(\mathbf{x})\right]^{T}$ is 438 obtained and used as input to our models.

\section{A. Transfer Learning Approach}

440 The proposed transfer learning approach has been inspired 441 by the fields of computer vision and natural language pro442 cessing [24], [25], where deep neural networks constitute the 443 state of the art for classification and prediction tasks. The core 444 idea of our approach is to train a neural network for the sig445 nal strength prediction task in a source domain (reference tilt 446 configuration) and then 'wisely' build a new neural network 447 to obtain fine-grained predictions in the target domain (tar448 get tilt configuration). The neural network architectures used 449 in our approach are FFNs, which are well-known for being 450 powerful nonlinear function approximators [38]. We opt for ${ }_{451}$ FFNs instead of more complex network architectures, such as ${ }_{452} \mathrm{CNNs}$ or recurrent neural networkss (RNNs) for two main 453 reasons. Firstly, from preliminary experimental results (see ${ }_{454}$ Figure 6), we observed that the achieved training and cross 455 validation losses are already very low and close to each other 456 for the problem at hand. Therefore, using a more complex architecture with the same limited amount of data available 457 for training could lead to a bigger gap between training and 458 cross validation, causing overfitting and thus worsening the 459 performance. Secondly, more complex architectures would 460 require more parameters and hyperparameters to be found, 461 causing an increased training time.

462

We use the Mean Square Error (MSE) as loss function, 463 which is the standard metric used in regression tasks. Here 464 the goal is to minimize the difference between the real and 465 predicted RSRP values. It is worth noting that the MSE is 466 well known for being sensitive to outliers, however this is 467 not a concern in this case since outliers have been removed 468 in previous preprocessing steps (see Section III-B). By using 469 FFNs as the basic building blocks of our architecture, the 470 flow of information only travels forward, and the layers of the 471 network are fully connected. Formally, FFNs learns a combi- 472 nation of parameters to find the best function approximation. 473 In our case, we aim at finding a set of parameters $\boldsymbol{\theta}$ for the 474 hidden layers and a set of parameters $w$ for the output layer 475 to estimate $\hat{s}(\mathbf{x}) \in \mathbb{R}^{q}$ for $\mathbf{x} \in \mathbb{R}^{p}$, as shown in Eq. (3):

$$
\hat{s}(\mathbf{x})=f(\mathbf{x} ; \boldsymbol{\theta}, \mathbf{w})=\boldsymbol{\phi}(\mathbf{x}, \boldsymbol{\theta})^{T} \mathbf{w}
$$

where $\phi: \mathbb{R}^{p} \rightarrow \mathbb{R}^{q}$, is a nonlinear transformation defining 478 the hidden layers, and parameters $\mathbf{w} \in \mathbb{R}^{q}$ map from $\boldsymbol{\phi}$ to the 479 desired output. Each input is represented by a tuple containing 480 distance, relative azimuth and relative angle (i.e., $\left.\left(d, \delta_{1}, \delta_{2}\right)\right){ }_{481}$ and each output is the RSRP value $\hat{s}(\mathbf{x})$ associated to a given 482 input. Therefore, $p=3$ and $q=1$ are the input and output 483 dimensions, respectively.

The proposed transfer learning approach is composed of the 485 following:

- $\mathcal{D}_{S}$ : source domain which consists of the feature space ${ }_{487}$ of the reference tilt configuration and its marginal prob- 488 ability distribution

489

- $\mathcal{D}_{T}$ : target domain which consists of the feature space of 490 the target tilt configuration and its marginal probability 491 distribution

492

- $\mathbf{M}_{S}=\hat{f}_{S}(\cdot)$ : an FFN with $n$ layers approximating the ${ }_{493}$ predictive function in the source domain $f_{S}(\cdot) \quad 494$

- $\mathbf{M}_{T}=\hat{f}_{T}(\cdot)$ : an FFN with $m$ layers approximating the 495 predictive function in the target domain $f_{T}(\cdot) \quad 496$

- $\left\{p_{1}, \ldots, p_{K}\right\}$ : the best combination of hyperparameters 497 shared by both FFNs associated with the source and target 498 domains respectively. ${ }^{1}$

499

The steps of our transfer learning algorithm are defined as 500 follows:

501

1) We select the source domain $\mathcal{D}_{S}$ and train $\mathbf{M}_{S}$ on 502 $\mathcal{D}_{S}$, finding the best combination of hyperparameters 503 $\left\{p_{1}, \ldots, p_{K}\right\}$. We use Bayesian optimization [39] since 504 it is an effective way of finding a suboptimal solution 505 in less time, when compared to random search [40], for 506 example. The problem of choosing the hyperparameters 507 is modeled as a sample of a Gaussian process (GP). We ${ }_{508}$ start with an initial combination of hyperparameters and ${ }_{509}$ dynamically update the searching space based on the 510

\footnotetext{
${ }^{1}$ For parameter transfer we assume that the models for source and target domains share a combination of hyperparameters.
} 


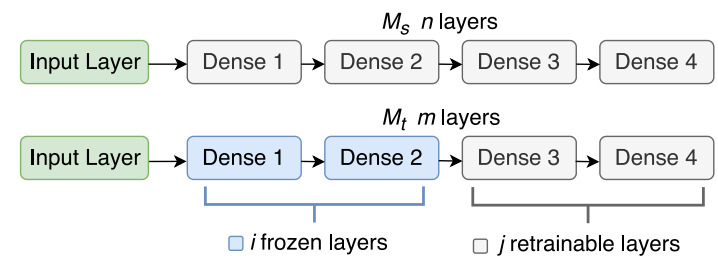

Fig. 4. Transfer learning model.

$511 \quad$ built surrogate probability model mapping from hyper512 parameters to the probability of a score on the objective 513 function (see Section V-B1 for numerical results). It is $514 \quad$ worth noting that the optimization process has been car515 ried out on $\mathcal{D}_{S}$ since we assume we do not have enough

548 due to its flexibility for implementing this transfer learning 549 approach and performing hyperparameters search. In total, the 550 training and testing phases of the two models do not last more 551 than two minutes. We use a laptop with 16 GB of RAM and 552 a 7th generation, Intel Core i7 processor.

\section{B. Baseline Methods}

${ }_{554}$ In this section, we describe the baseline methods used to 555 benchmark our work: Heuristic $(\mathrm{H})$ using data provided by 556 antenna manufacturer as well as k-Nearest Neighbors (k-NN) 557 and Random Forest (RF), which performed the best for the 558 task at hand in our previous work [1].
1) Heuristic: This is the simplest baseline method, where 559 the predicted values are extracted from the data sheets pro- 560 vided by the antenna manufacturer. Given a set of locations at ${ }_{561}$ a given tilt configuration, for each sample we create the fea- 562 ture vector by calculating distance, relative angle and relative 563 azimuth (Section IV). In a second step, we use the data sheets 564 provided by the antenna manufacturer to extract the antenna 565 gain on the vertical and horizontal planes. Finally, we apply 566 the path loss model to calculate the predicted values. Formally, ${ }_{567}$ the process is defined as follows:

1. Given $\mathcal{M}_{h}$ as the set of location indexes where measure- ${ }_{569}$ ments for the considered base station running configu- 570 ration $h$ have been taken, we calculate for each location 571 $\mathbf{x} \in \mathcal{M}_{h}$ a tuple of values $\left(d, \delta_{1}, \delta_{2}\right)$. Then we create 572 the feature vector $\mathbf{z}:=\left[d(\mathbf{x}), \delta_{1}(\mathbf{x}, h), \delta_{2}(\mathbf{x})\right]^{T}$ as shown ${ }_{573}$ in Section IV.

574

2. Let $\eta(\mathbf{x})$ and $\gamma(\mathbf{x})$ be the horizontal and vertical gain 575 of the antenna in $\mathrm{dB}$, respectively, as taken from the 576 manufacturer antenna sheets. Given the known position 577 $\mathbf{x}$, we formally define $\Delta(\mathbf{x})$ as:

$$
\Delta(\mathbf{x})=\eta(\mathbf{x})+\gamma(\mathbf{x})
$$

3. Given $\Delta(\mathrm{x})$, we use the path loss model to generate the 580 labels, $\hat{s}(\mathbf{x})$, by applying the following:

$$
\hat{s}(\mathbf{x})=\phi_{0}+\phi_{1} 10 \log (d(\mathbf{x}))-\Delta(\mathbf{x}),
$$

where $\phi_{0}$ and $\phi_{1}$, similar to [10], are the linear regres- ${ }_{583}$ sion coefficients calculated for the reference dataset. $\quad 584$

2) k-Nearest Neighbors With Inverse Distance Weighting: 585 This technique is one of the simplest multivariate interpo- 586 lation methods which extends the classical nearest neighbor 587 approach [43]. We apply the technique on the same feature 588 vector $\mathbf{z}(\mathbf{x}):=\left[d(\mathbf{x}), \delta_{1}(\mathbf{x}), \delta_{2}(\mathbf{x})\right]^{T}$ as defined in the above- 589 mentioned Heuristic approach. It predicts the signal at an 590 unknown target location $\mathbf{x}$ (corresponding to a feature vector 591 $\mathbf{z}(\mathbf{x}))$ as a weighted average of the signals at the $k$ locations 592 with the closest distances calculated based on feature vectors. 593

$$
\hat{s}(\mathbf{z})=\sum_{i \in \mathcal{M}(\mathbf{z})} \omega_{i} s\left(\mathbf{z}_{i}\right)
$$

The set $\mathcal{M}(\mathbf{z})$ includes the feature vectors which are the clos- 595 est to the unknown target vector $\mathbf{z}$, with cardinality $|\mathcal{M}|=k$. 596 Weights $\omega_{i}$ are chosen to be inversely proportional to the dis- 597 tance $d\left(\mathbf{z}_{i}, \mathbf{z}\right)$ and their sum is normalized to one, using the 598 equation below:

$$
\omega_{i}=\frac{d\left(\mathbf{z}_{i}, \mathbf{z}\right)^{-1}}{\sum_{j \in \mathcal{M}(\mathbf{z})} d\left(\mathbf{z}_{j}, \mathbf{z}\right)^{-1}} .
$$

3) Random Forest: Is one of the ensemble methods used 601 for classification and regression purposes. The algorithm was 602 introduced by Ho [44] in 1995, and later extended by Breiman 603 and Cutler [45], it uses the idea of bagging to perform 604 predictions. During the process several trees are grown inde- 605 pendently using different bootstrapped samples of the data and 606 majority voting or averaging is used for the final prediction. 607 In contrast to traditional trees, the variable used to perform 608 the split in each node is chosen randomly from a set of pre- 609 dictors [45]. RF is known to sometimes outperform other 610 
611 machine learning techniques, such as neural networks, due to 612 its resistance to overfitting [46].

\section{EXPERIMENTS}

614 In this section, we describe the set of experiments carried 615 out. We compare the prediction error of our transfer learning 616 method (i.e., DNN T in Section IV-A) against the baseline 617 methods (i.e., H, k-NN, RF in Section IV-B). In the following, 618 the suffix $\mathrm{T}$ is used to denote the transfer learning approach 619 (i.e., DNN T). Similarly, the suffix $\mathrm{S}$ is used to denote the 620 methods that do not use transfer learning (i.e., H S, k-NN S, ${ }_{621} \mathrm{RF}$ S and DNN S). It is worth noting that DNN T is trained 622 on data from a different tilt configuration (source domain) 623 whereas H S, k-NN S, RF S and DNN S are trained on data 624 from the same tilt configuration (target domain). In this way, 625 we compare the performance of the proposed transfer learning 626 solution against the performance of traditional machine learn627 ing solutions to reveal the scenarios where a transfer learning 628 solution is preferred. We also train a model on the source 629 domain and apply it to the target domain without the retrain630 ing and fine tuning step. This last approach is referred as DNN ${ }_{631}$ BS. It does not require data from the target domain since no 632 retraining is performed. In this case, the purpose is carrying out 633 comparisons against the transfer learning solution to evaluate 634 the real need for the retraining and fine tuning step.

635 We carry out two different sets of experiments that differ in 636 the way the source domain is built. In Section V-C, the source 637 domain consists of measurements from a single tilt config638 uration, which differs to the one used for target domain. In 639 Section V-D we augment the source domain by adding mea640 surements from other available tilt configurations of the same 641 PCI. In both cases, we analyze the impact on the performance 642 when a limited amount of data from the target domain is avail643 able in the training phase. We study two strategies to select 644 data from the target domain: (i) uniformly distributed in the 645 reference area or ii) non-uniformly distributed according to 646 a predefined sampling strategy (i.e., different distance ranges 647 from the antenna location).

648 For each tilt configuration the amount of data available is 649 about 600 measurements. In all the experiments, the data is 650 divided into training, cross validation and test sets. We use $65180 \%$ of samples for training, $10 \%$ for cross validation and $65210 \%$ for testing. We vary the quantity of data taken from the 653 target domain for training or fine tuning. For the DNN T, this 654 is the number of samples used to train and fine tune $\mathbf{M}_{T}$. For 655 the k-NN S, RF S and DNN S this is the quantity of data avail656 able for training a model on the target domain using data from 657 the same target domain. In contrast, H S does not need train658 ing data. One of the main objectives is to map the amount 659 of labeled data required from the target domain and corre${ }_{660}$ sponding performance, assessed in terms of Mean Absolute 661 Percentage Error (MAPE), which is defined as:

$$
662 \quad \mathrm{MAPE}=\frac{100}{k} \sum_{i=0}^{k-1}\left|\frac{s_{i}-\hat{s_{i}}}{s_{i}}\right| \text {, }
$$

663 where $k$ is the number of target positions in the testing dataset.

\section{A. Domain Distance}

664

Since the performance of the transfer learning approach 665 depends on the similarity between the training and testing sets 666 on the target domain, we introduce a measure of the degree of 667 similarity between datasets which is then used throughout this 668 section. We quantify similarity in terms of Kullback-Leibler 669 (KL) divergence index [47], which measures the relative 670 entropy of a given probability distribution with respect to 671 another one. Given two reference datasets, one used for train- 672 ing and one used for testing (both in the target domain), we 673 derive the KL divergence indexes of the probability distribu- 674 tions of the logarithm of the distance $(d)$, relative angle $\left(\delta_{1}\right) 675$ and relative azimuth $\left(\delta_{2}\right)$ of the two datasets. Formally, the 676 symmetric KL divergence index of the distance probability 677 distributions is given by:

$$
\begin{aligned}
S D_{K L}(d)= & \sum_{i=1}^{k} P_{d}^{(\mathrm{tr})}(i) \log \frac{P_{d}^{(\mathrm{tr})}(i)}{P_{d}^{(\mathrm{te})}(i)} \\
& +\sum_{i=1}^{k} P_{d}^{(\mathrm{te})}(i) \log \frac{P_{d}^{(\mathrm{te})}(i)}{P_{d}^{(\mathrm{tr})}(i)}
\end{aligned}
$$

where $P_{d}^{(\operatorname{tr})}(i)$ and $P_{d}^{(\text {te })}(i)$ with $i=1 \ldots k$ define the dis- 681 crete probability distributions of the distance in the training 682 and testing sets of the target domain, respectively and $k$ is 683 the amount of bins used to estimate either $P_{d}^{(\mathrm{tr})}$ or $P_{d}^{(\mathrm{te})} \cdot 684$ Similar definitions hold for the KL divergence indexes related 685 to the relative angle $\delta_{1}$ and relative azimuth $\delta_{2}$. Finally, to 686 give a more succinct representation of domain similarity, we 687 introduce the Domain Distance (DD) measure by summing the 688 three indexes together:

$$
\mathrm{DD}=S D_{K L}(d)+S D_{K L}\left(\delta_{1}\right)+S D_{K L}\left(\delta_{2}\right) . \quad \text { (10) } 690
$$

Figure 5 shows the average DD across PCIs for all the pos- 691 sible combinations of training and testing sets on the target 692 domains and the amount of points from the target domain used 693 for training. The solid curve in Figure 5 shows the DD when 694 the available samples taken from the target domain for training 695 or fine tuning are uniformly sampled in the reference area. The 696 dashed curve in Figure 5 shows the DD when the samples are 697 taken between $300 \mathrm{~m}$ and $600 \mathrm{~m}$ of distance from the antenna 698 location. Smaller DD values indicate higher domain similarity 699 and vice versa. For instance, when the samples are uniformly 700 distributed the similarity between training and testing sets in 701 the target domain is higher, which makes the DD values lower, 702 i.e., they range from 0.8 to 1.3 (see Figure 5 solid curve). In 703 contrast, when the available measurements are located at a 704 certain distance range from the antenna (i.e., 300 to $600 \mathrm{~m}$ ), 705 similarity is lower, meaning the DD values are higher ranging 706 from 1.5 to more that 2 (see Figure 5 dashed curve).

707

\section{B. Hyperparameter Search}

708

Hyperparameters are chosen in a hybrid manner by using a 709 mixture of Bayesian optimization and manual fine tuning. $\quad 710$

1) Hyperparameter Search on $\mathcal{D}_{S}$ : Bayesian optimization 711 requires, as starting point, a network architecture that con- 712 verges. It also requires that the hyperparameters search space 713 


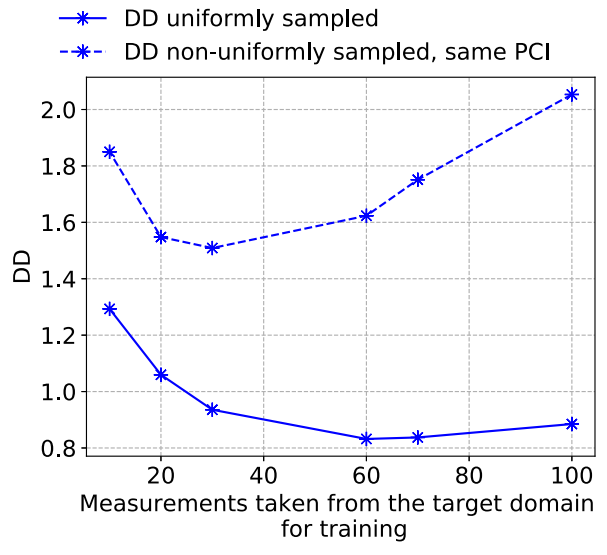

Fig. 5. Domain distance.

TABLE I

MAPE FOR VARIATIONS IN THE AMOUNT OF LAYERS AND HIDDEN UNITS FOR PCI 1, TILT 2, 3 AND 6

\begin{tabular}{|c|c|}
\hline $\mathcal{D}_{S}$ Network architecture & Avg. across Tilts \\
\hline $4,10,4,1$ & 4.30 \\
\hline $4,10,4,4,1$ & 11.94 \\
\hline $4,10,1$ & 5.58 \\
\hline $4,20,8,1$ & 6.82 \\
\hline $4,5,2,1$ & 5.59 \\
\hline
\end{tabular}

714 is specified. We begin with an architecture that contains 4 lay715 ers with 4, 10, 4 and 1 hidden units, respectively, $1 e-3$ as the 716 initial learning rate and $\mathrm{ReLu}$ as the initial activation function. 717 The activation function search space contains Sigmoid and ${ }_{718} \mathrm{ReLu}$, and the learning rate search space goes from $1 e-7$ to $719 l e-1$. After 50 iterations the process converges and we find 720 out that for all the tilt configurations and PCIs the best learn721 ing rate is approximately 0.099 , and the choice of activation 722 function that leads to the minimal error is Sigmoid. During this ${ }_{723}$ process, the model is shown to achieve good performance in 724 the source domain. Figure 6 shows the training and cross vali725 dation errors for PCI 1 and tilts 2, 3 and 6. Comparable results 726 are achieved for the rest of the PCIs and tested tilt combina727 tions. It can be observed that the training and cross validation 728 errors decrease dramatically during the first 150 epochs for tilts 7292 and 3 and the first 20 epochs for tilt 6 . After this they keep 730 decreasing steadily, becoming very close to 0 , which means 731 the chosen architecture fits the data coming from $\mathcal{D}_{S}$. We 732 note that both errors are close to each other, meaning that our 733 model is not overfitting. Once the learning rate and activa734 tion functions are chosen, we carry out experiments on $\mathcal{D}_{S}$, 735 increasing and decreasing the model complexity by adding and 736 removing layers and hidden units, respectively. Table I shows 737 the MAPE obtained leveraging the tested architectures. The 738 reported MAPE is an average across all the available PCIs 739 and all the available combinations of tilt configurations as 740 source and target domain. It can be observed that increasing or 741 decreasing complexity worsens the performance for all the tilt 742 configurations on average, therefore the initial combination of 7434 layers containing 4, 10, 4 and 1 hidden units respectively is 744 the one that leads to the best performance. Table II summarizes 745 the best combination of hyperparameters found by a mixture 746 of Bayesian and manual optimization.
TABLE II

HYPERPARAMETERS FOUND BY BAYESIAN AND MANUAL OPTIMIZATION

\begin{tabular}{|c|c|}
\hline Number of epochs & 500 \\
\hline Batch size & 128 \\
\hline Number of inputs & 3 \\
\hline Number of layers & 4 \\
\hline Hidden units per layer & $4,10,4,1$ \\
\hline Activation function & Sigmoid \\
\hline Optimizer & Adam \\
\hline Learning rate & 0.099 \\
\hline
\end{tabular}

2) Frozen and Re-Trainable Layers: As explained in 747 Section IV-A, the amount of layers to freeze and retrain is 748 chosen through an empirical approach, trying all possible com- 749 binations and choosing the best one. Figure 7 reports the 750 MAPE averaged across all the PCIs and all the possible com- 751 binations of tilt configurations as source and target domain 752 when using different numbers of layers to freeze, $i$ and the 753 number of retrainable layers, $j$. DNN T method indicates that 754 the weights in the retrainable layers of $\mathcal{M}_{T}$ are randomly ${ }_{755}$ initialized, whereas the DNN T W indicates that the weights 756 in the retrainable layers are initialized with the weights from 757 $\mathcal{M}_{S}$ after training. We use $\mathrm{F}$ and $\mathrm{R}$ to denote the number of ${ }_{758}$ layers to freeze and retrain on $\mathcal{M}_{T}$, respectively. We select 759 $i=2$ and $j=2$ (i.e., DNN T 2F 2R), since it is the combination 760 of values that leads to the best MAPE on $\mathcal{D}_{T}$.

\section{Single Tilt Transfer}

762

We use a dataset obtained under a given tilt setting (source 763 domain) to predict the performance of the same antenna under 764 a different tilt configuration (target domain). In particular, 765 we consider two different scenarios: when the data available 766 from the target domain is limited and sampled uniformly (see 767 Section V-C1) and when the data is still limited but sampled 768 according certain criteria, for instance at a given range of the 769 antenna location (see Section V-C2).

1) Limited and Uniformly Sampled Measurements: In this 771 case measurements represent a wide range of relative dis- 772 tances, azimuth and RSRP values. The amount of instances 773 taken for training or fine tuning varies between 0 to 100 . 774 Figure 8 shows the average MAPE across all the PCIs and pos- 775 sible pairs of training and testing tilt combinations, obtained 776 by the different prediction approaches described in Section IV. 777 We can draw the following conclusions:

778

- All the machine learning methods (i.e., k-NN S, RF S, 779 DNN S, DNN T) outperform the heuristic approach (i.e., 780 H S) for any number of instances taken from the target 781 domain for training or fine tuning. Therefore, the machine 782 learning algorithms trained on real data are more effec- 783 tive at capturing the non linearity of RSRP values than 784 the heuristic approach which uses the path loss model 785 to extract RSRP values from the sheets provided by the 786 antenna manufacturer.

787

- The prediction error is impacted by the amount of sam- 788 ples taken from the target domain. In particular, the 789 amount of data taken from the target domain can be 790 decreased by up to $90 \%$, if we consider an initial amount 791 of 590 instances taken for training or fine tuning, with 792 


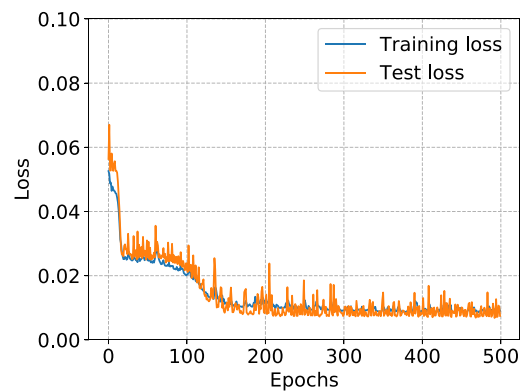

(a) Tilt 2

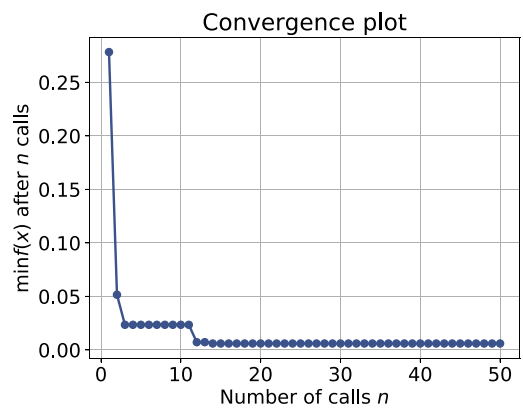

(d) Tilt 2

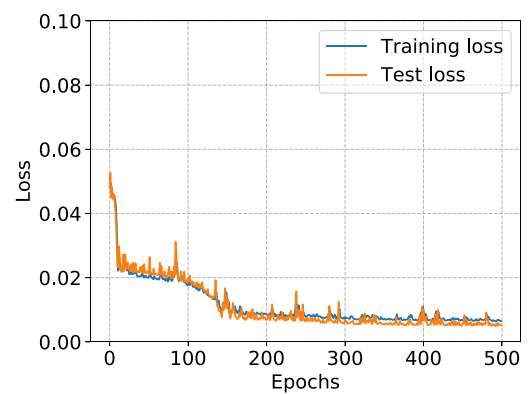

(b) Tilt 3

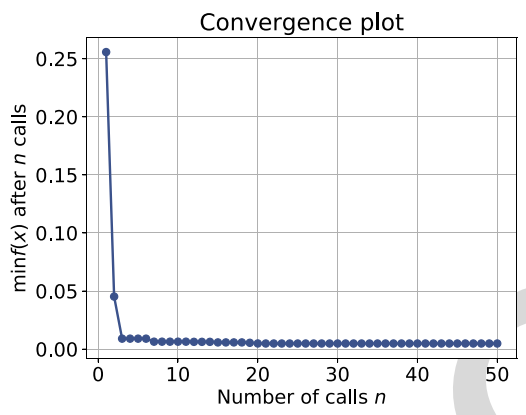

(e) Tilt 3

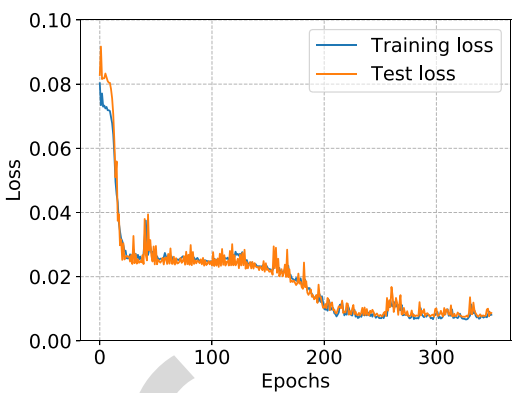

(c) Tilt 6

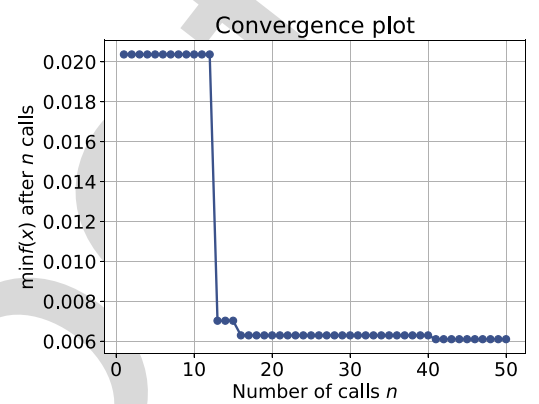

(f) Tilt 6

Fig. 6. Training curves and Bayesian convergence on the source domain, PCI 1.

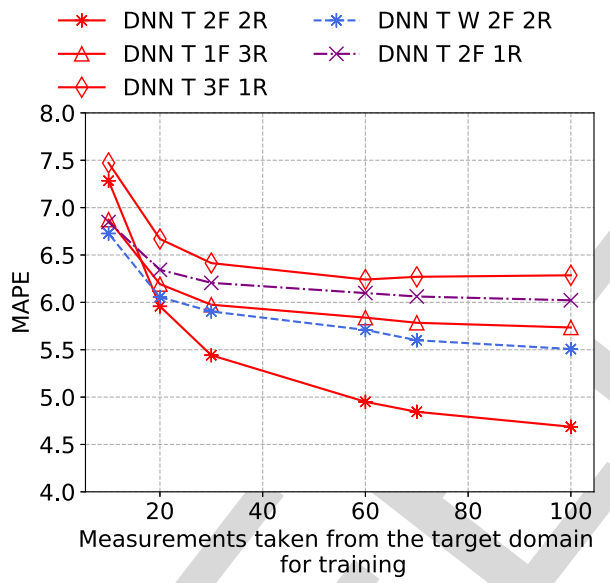

Fig. 7. MAPE for the different values of frozen $(\mathrm{F})$ and retrainable $(\mathrm{R})$ layers using random or source domain weights initialization.

a maximum increase in error rate of $2 \%$ for the transfer learning approach. It is worth noting that if no data is taken from the target domain, the transfer learning approaches must be used under the assumptions of traditional machine learning, where source and target domain are similar. As this is not the case, DNN T outperforms DNN BS when the amount of instances taken for training is more than 20. This justifies the need to model our problem under the framework of transfer learning in order to decrease the prediction error.

- The transfer learning approach (i.e., DNN T) outperforms the methods that use data from the same tilt configuration (k-NN S, RF S and DNN S) for training, when

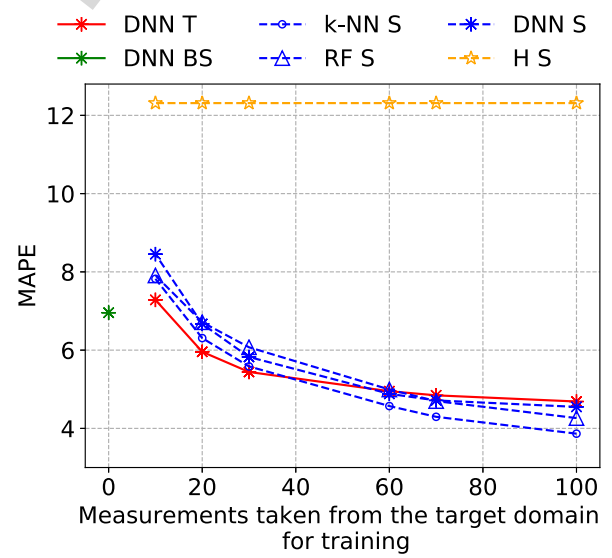

Fig. 8. MAPE when training or fine tuning on uniformly sampled measurements.

the amount of samples taken from the testing set is less 806 than 40 instances out of 590. This is because, transfer 807 learning approaches better capture the physical properties 808 of antenna propagation. Thus, being more robust when 809 information is missing from the cross-domain.

810

- When the number of data samples is larger than 60, 811 transfer learning performs worse than the non-transfer 812 methods. This indicates, more than 60 points chosen uni- 813 formly for training a model are enough to capture all 814 the possible patterns (different RSRP values) in a given 815 radio map while achieving good prediction error (see DD 816 values uniformly sampled curve in Figure 5 for more 817 than 60 measurements). In contrast, if less than 60 points 818 


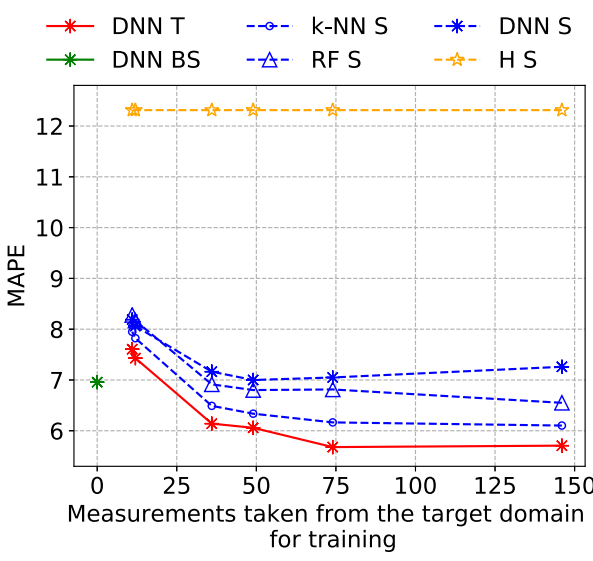

(a) $0-300 \mathrm{~m}$

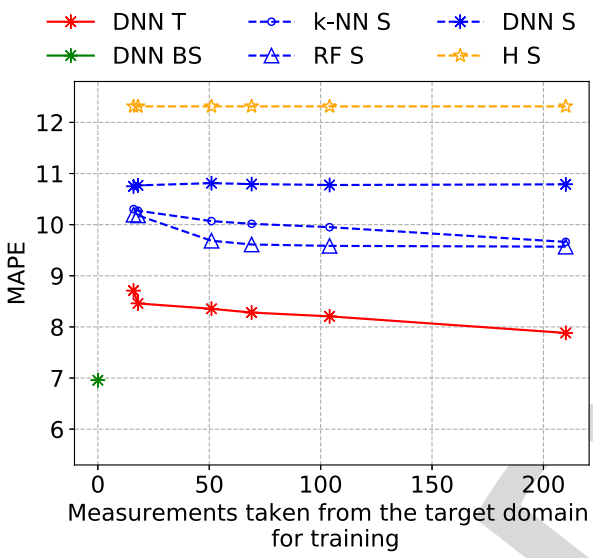

(c) Above $600 \mathrm{~m}$

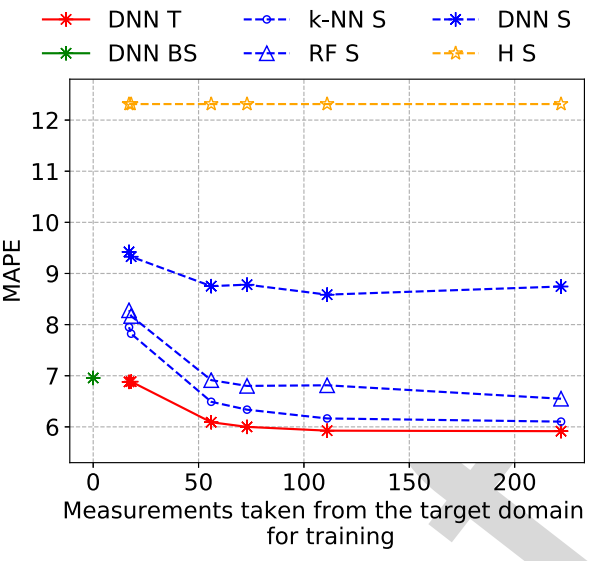

(b) $300-600 \mathrm{~m}$

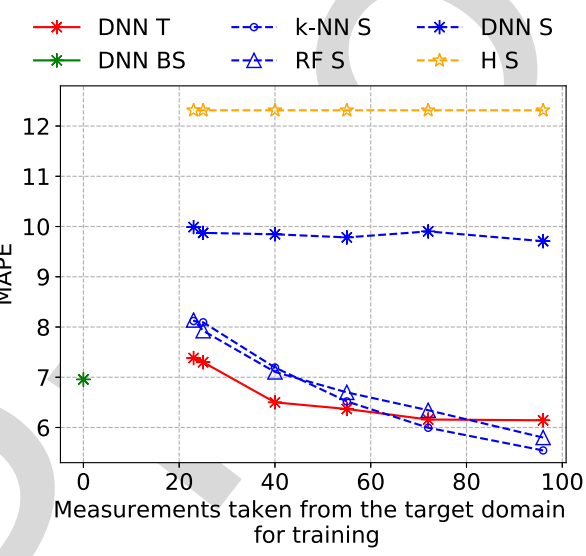

(d) Probability $0.1,0.1$ and 0.8

Fig. 9. MAPE when training or fine tuning on non-uniformly sampled measurements.

826 We define different antenna distance ranges since we assume 827 to have available only measurements collected in one of those 828 locations. Figure 9 shows the obtained average MAPE across 829 all PCIs and all possible combinations of training and test830 ing tilts. Figures 9(a), 9(b) and 9(c) show the average MAPE 831 for measurements collected between 0 to 300,300 to 600 , 832 and more than $600 \mathrm{~m}$ from the antenna location, respectively. 83 In addition, we consider in Figure 9(d), the case where we ${ }_{834}$ take points from all of the three ranges with probability 0.1 , 8350.1 and 0.8 , respectively. This set of experiments, is moti836 vated by the fact that in a realistic scenario points at a given 837 distance range might be the only ones available to carry out 838 predictions. For instance, in some areas it might not be possi839 ble to take measurements due to the existence of obstacles or 840 private properties. In other cases there might be budget con841 straints (both in terms of resources and time) which do not 842 allow for an extensive measurement campaign of the whole 843 area. These scenarios are particularly challenging, because tra844 ditional methods do not work at their best. Therefore we focus on these to highlight the benefits of transfer learning. To study 845 these cases, we consider two possible options: (i) we use the 846 available points as the training set to carry out predictions 847 under the same tilt configuration (i.e., H S, k-NN S, RF S, 848 DNN S) or (ii) we use the available points as part of the 849 retraining step in the transfer learning pipeline (i.e., DNN T). 850 In both cases, the model output is the predicted radio map for ${ }^{851}$ the whole area.

We can draw the following conclusions:

852

- As before, all the machine learning methods (i.e., $\mathrm{H} \mathrm{S}$, 954 k-NN S, RF S, DNN S and DNN T) outperform the 855 heuristic approach (i.e., H S) and are impacted by the 856 amount of instances taken from the target domain for ${ }^{857}$ training or fine tuning the models. 858

- The prediction error is never more than $2 \%$ higher than 859 when using uniformly sampled data. An increase in error 860 is expected since training and testing sets in the target 861 domain are more dissimilar than when samples are taken 862 uniformly (see DD values in Figure 5). However, depend- 863 ing on the application and data restriction when collecting 864 samples, non-uniformly distributed data could still be 865 used to carry out predictions when uniformly sampled 866 data is not available.

- The transfer learning approach (i.e., DNN T) outperforms 868 the methods that use data from the same tilt configuration 869 (i.e., k-NN S, RF S and DNN S) to carry out predictions 870 
TABLE III

DD Values Before and After Data Augmentation

\begin{tabular}{|c|c|c|}
\hline Algorithm & DD & $\mathcal{D}_{S}$ Number of samples \\
\hline No augmentation & 1.15 & 600 \\
\hline Augmentation same PCI & 1.39 & 1200 \\
\hline Augmentation other PCIs & 3.53 & 4800 \\
\hline
\end{tabular}
882 able to outperform other methods when using real measure883 ments for both uniformly and non-uniformly sampled data. It 884 has benefits compared to the benchmark methods in both of 885 the following situations: (1) when the available samples of the 886 target domain data are sampled uniformly, but the number of 887 the samples is limited, and (2) when the available measure888 ments used for training or fine tuning on the target domain 889 are non-uniformly sampled. Moreover, considerable accuracy 890 gains are achieved when augmenting the source domain with ${ }_{891}$ data coming from other available tilt configurations of the 892 same antenna. This is discussed in the next section.

\section{D. Tilt Augmentation Transfer}

894 Data augmentation has been shown to be successful in the 895 area of computer vision. By augmenting an existing dataset 896 with new data that follows the same distribution as the data 897 used for training, overfitting can be reduced [48]. In our case, 898 we take inspiration from this idea and we augment the source 899 domain by adding data from other available tilt configurations 900 within the same PCI (i.e., suffix A SP on the graphs below) and 901 from different PCIs (i.e., suffix A OP). We map the obtained 902 MAPE to the DD to analyze the cases where data augmenta903 tion improves performance. Table III shows the DD between 904 the training set in $\mathcal{D}_{S}$ and the test set in $\mathcal{D}_{T}$ both before 905 data augmentation and after data augmentation. Data augmen906 tation is performed by either adding data from the same PCI 907 or adding data from the same and different PCIs. Table III 908 also shows the total amount of training samples used in each ${ }_{909}$ case in $\mathcal{D}_{S}$. It can be noted that, DD values are much higher 910 when using data from different PCIs than in the rest of the 911 cases. This is because adding data from a different PCI will 912 increase the difference between the training set in $\mathcal{D}_{S}$ and the ${ }_{913}$ test set in $\mathcal{D}_{T}$, therefore overfitting will be increased in $\mathcal{D}_{T}$. 914 However, the degree of similarity between radio maps coming 915 from the same PCI is higher, thus adding data with a greater 916 similarity to the training set in $\mathcal{D}_{S}$ and test set in $\mathcal{D}_{T}$ can help 917 to reduce overfitting and improve accuracy. We evaluate the 918 gains of performing transfer learning from a bigger and more 919 diverse source domain.

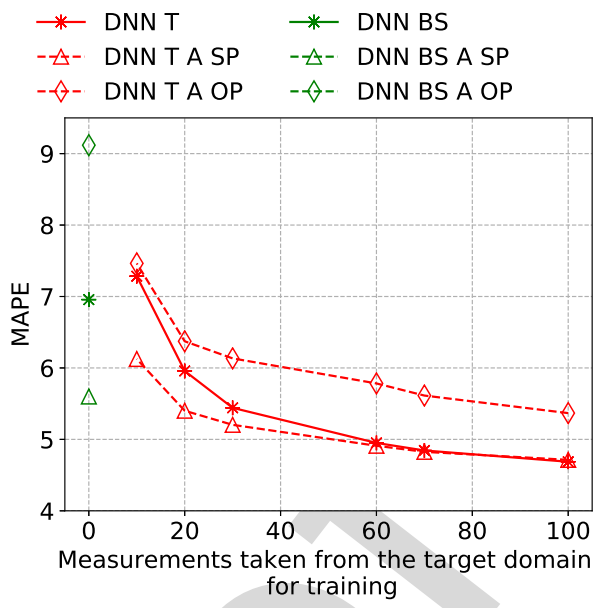

(a) Limited uniformly sampled data

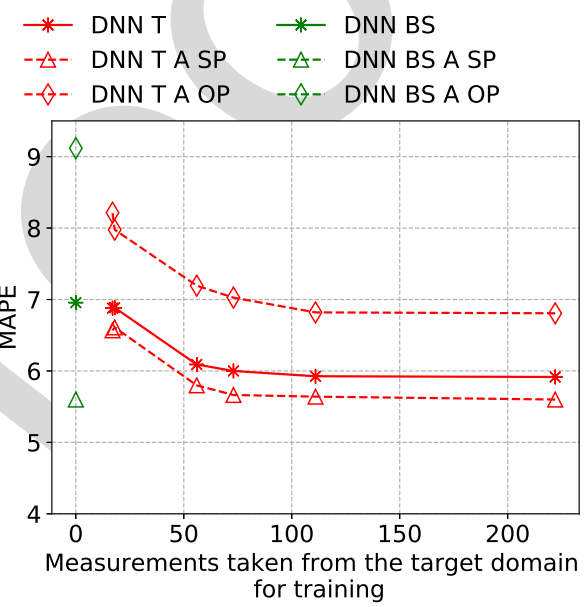

(b) Limited non-uniformly sampled data

Fig. 10. MAPE with and without data augmentation.

Figure 10 shows the average MAPE across all the PCIs 920 and pairs of training and testing tilt combinations possible ${ }_{921}$ when performing data augmentation on the source domain. 922 Figure 10(a) shows the average MAPE when the instances ${ }_{923}$ taken from the target domain for training or fine tuning are ${ }_{924}$ limited and sampled uniformly. In contrast, Figure 10(b) shows 925 the average MAPE for cases when the measurements taken 926 from the target domain were collected at a distance range ${ }_{927}$ from the antenna between 300 and $600 \mathrm{~m}$. We can draw the ${ }_{928}$ following conclusions:

- When using data augmentation on the source domain, the 930 prediction error decreases by more than a $1 \%$ when the ${ }_{931}$ amount of instances taken from the target domain varies 932 between 10 and 40 (see Figure 10(a)).

- In Figure 10(b) we can also observe a performance 934 improvement when compared to the performance 935 achieved without augmenting the source domain.

936

- In both cases, the performance improvement can be ${ }_{937}$ explained by the fact that data augmentation reduces over- 938 fitting. Figure 11 shows the training and cross validation 939 curves for PCI 1 when Tilts 6 and 2 are used as source 940 and target domains, respectively. Figures 11(a) and 11(b) 941 illustrate the training and cross validation losses without 942 


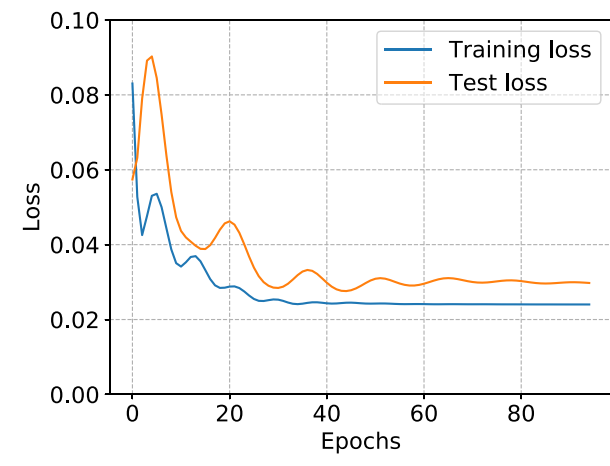

(a) Without data augmentation

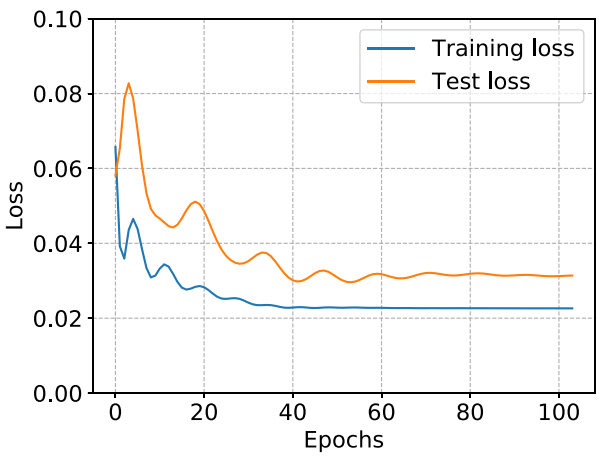

(c) Without data augmentation

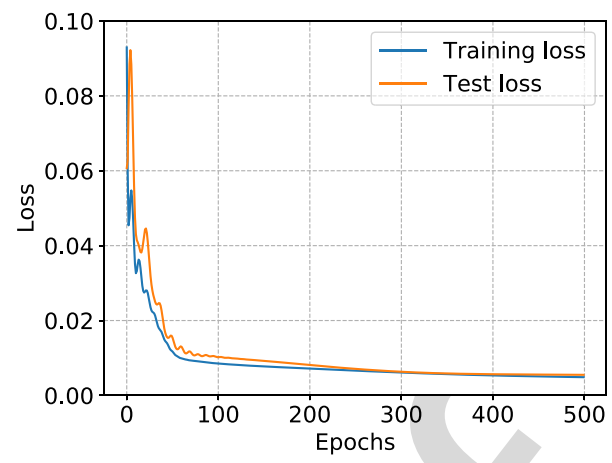

(b) With data augmentation

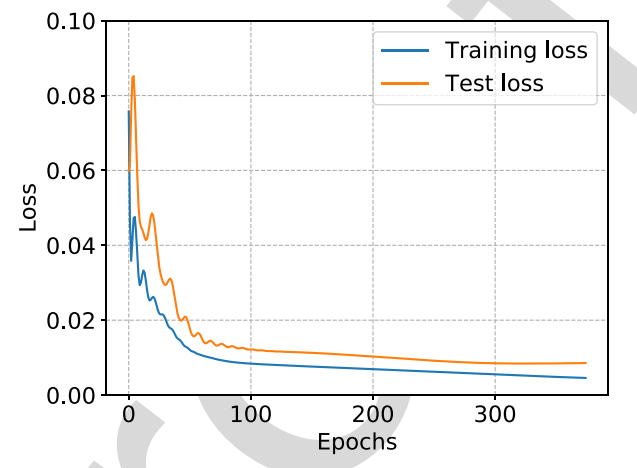

(d) With data augmentation

Fig. 11. Training curves on the target domain, PCI 1 and Tilts 6 and 2: (a), (b) Limited uniformly sampled data, (c), (d) Limited non-uniformly sampled data. 962 signal strength in the downlink of a real LTE network, where 963 the antennas can be tuned to operate with different antenna tilt 964 configurations. Different approaches were considered as candi965 dates for predicting the signal strength. All of them were based 966 on refined features related to propagation and antenna config967 uration. As opposed to other works in the field of radio map 968 inference, we studied the quality of prediction of the afore969 mentioned approaches when the datasets used for training and 970 testing are related, but not sampled from the same distribution. ${ }_{971}$ We observed that the performance of the predictive models is dependent on the amount of data taken from the testing domain 972 for training or fine tuning. Furthermore, the proposed transfer 973 learning algorithms are shown to be more efficient in cases 974 where the amount of data available from the target tilt config- 975 uration is very limited, or available at different distance ranges 976 from the antenna location. Finally, we have shown how aug- 977 menting data from the source domain by adding data available 978 from other tilts configurations of the same antenna improves 979 the performance of the proposed transfer learning approaches. 980 Augmenting the source domain decreases the prediction error ${ }_{981}$ by $1 \%$ when the data available from the target domain for 982 training or fine tuning is limited, or at a distance range between 983 300 and $600 \mathrm{~m}$ from the antenna location.

\section{REFERENCES}

[1] C. Parera, A. E. C. Redondi, M. Cesana, Q. Liao, L. Ewe, and C. Tatino, 986 "Transferring knowledge for tilt-dependent radio map prediction," in 987 Proc. IEEE Wireless Commun. Netw. Conf. (WCNC), 2018, pp. 1-6. 988

[2] C. Jiang, H. Zhang, Y. Ren, Z. Han, K.-C. Chen, and L. Hanzo, 989 "Machine learning paradigms for next-generation wireless networks," 990 IEEE Trans. Wireless Commun., vol. 24, no. 2, pp. 98-105, Apr. 2017. 991

[3] M. Danneberg, J. Holfeld, M. Grieger, M. Amro, and G. Fettweis, 992 "Field trial evaluation of UE specific antenna downtilt in an LTE down- 993 link," in Proc. IEEE Int. ITG Workshop Smart Antennas (WSA), 2012, 994 pp. 274-280.

[4] S. J. Pan and Q. Yang, "A survey on transfer learning," IEEE Trans. 996 Knowl. Data Eng., vol. 22, no. 10, pp. 1345-1359, Oct. 2010.

[5] J. Devlin, M.-W. Chang, K. Lee, and K. Toutanova, "BERT: Pre-training 998 of deep bidirectional transformers for language understanding," in Proc. 999 Conf. North Amer. Ch. Assoc. Comput. Linguist. Human Lang. Technol., 1000 2019, pp. 4171-4186.

1001

[6] J. Howard and S. Ruder, "Universal language model fine-tuning for text 1002 classification," in Proc. 56th Annu. Meeting Assoc. Comput. Linguist., 1003 2018, pp. 328-339. 
[7] J. Moysen, L. Giupponi, and J. Mangues-Bafalluy, "A mobile network planning tool based on data analytics," Mobile Inf. Syst., vol. 2017, Nov. 2017, Art. no. 6740585.

[8] H. C. Nguyen et al., "Validation of tilt gain under realistic path loss model and network scenario," in Proc. IEEE Veh. Technol. Conf. (VTC), 2013, pp. 1-5.

[9] C. Phillips, D. Sicker, and D. Grunwald, "A survey of wireless path loss prediction and coverage mapping methods," IEEE Commun. Surveys Tuts., vol. 15, no. 1, pp. 255-270, 1st Quart., 2013.

[10] I. Rodriguez et al., "A geometrical-based vertical gain correction for signal strength prediction of downtilted base station antennas in urban areas," in Proc. IEEE Veh. Technol. Conf. (VTC), 2012, pp. 1-5.

11] D. W. Kifle, B. Wegmann, I. Viering, and A. Klein, "Impact of antenna tilting on propagation shadowing model," in Proc. IEEE Veh. Technol. Conf. (VTC), 2013, pp. 1-5.

[12] "Evolved universal terrestrial radio access (E-UTRA); Further advancements for (E-UTRA) physical layer aspects," 3GPP, Sophia Antipolis, France, Rep. TR 36.814, 2006.

13] K. Weiss, T. M. Khoshgoftaar, and D. Wang, "A survey of transfer learning," J. Big Data, vol. 3, no. 1, p. 9, 2016.

14] M. E. Taylor and P. Stone, "Transfer learning for reinforcement learning domains: A survey," J. Mach. Learn. Res., vol. 10, no. 7, pp. 1633-1685, 2009.

15] J. Jiang and C. Zhai, "Instance weighting for domain adaptation in NLP," in Proc. 45th Annu. Meeting Assoc. Comput. Linguist., 2007, pp. 264-271.

16] R. Chattopadhyay, Q. Sun, W. Fan, I. Davidson, S. Panchanathan, and J. Ye, "Multisource domain adaptation and its application to early detection of fatigue," ACM Trans. Knowl. Disc. Data, vol. 6, no. 4, p. 18, 2012.

17] L. Duan, D. Xu, and I. W.-H. Tsang, "Domain adaptation from multiple sources: A domain-dependent regularization approach," IEEE Trans. Neural Netw. Learn. Syst., vol. 23, no. 3, pp. 504-518, Mar. 2012.

18] S.-I. Lee, V. Chatalbashev, D. Vickrey, and D. Koller, "Learning a metalevel prior for feature relevance from multiple related tasks," in Proc. ACM 24th Int. Conf. Mach. Learn., 2007, pp. 489-496.

19] M. Long, J. Wang, G. Ding, S. J. Pan, and S. Y. Philip, "Adaptation regularization: A general framework for transfer learning," IEEE Trans. Knowl. Data Eng., vol. 26, no. 5, pp. 1076-1089, May 2014.

20] J. Gao, W. Fan, J. Jiang, and J. Han, "Knowledge transfer via multiple model local structure mapping," in Proc. 14th ACM SIGKDD Int. Conf. Knowl. Disc. Data Mining, 2008, pp. 283-291.

21] T. Tommasi, F. Orabona, and B. Caputo, "Learning categories from few examples with multi model knowledge transfer," IEEE Trans. Pattern Anal. Mach. Intell., vol. 36, no. 5, pp. 928-941, May 2014.

22] L. Mihalkova, T. Huynh, and R. J. Mooney, "Mapping and revising Markov logic networks for transfer learning," in Proc. AAAI 22nd Nat. Conf. Artif. Intell., 2007, pp. 608-614.

[23] J. Davis and P. Domingos, "Deep transfer via second-order Markov logic," in Proc. 26th Annu. Int. Conf. Mach. Learn., 2009, pp. 217-224.

24] M. Oquab, L. Bottou, I. Laptev, and J. Sivic, "Learning and transferring mid-level image representations using convolutional neural networks," in Proc. IEEE Conf. Comput. Vis. Pattern Recognit., 2014, pp. 1717-1724.

[25] D. C. Cireşan, U. Meier, and J. Schmidhuber, "Transfer learning for Latin and Chinese characters with deep neural networks," in Proc. IEEE Int. Joint Conf. Neur. Netw. (IJCNN), 2012, pp. 1-6.

[26] M. Chen, U. Challita, W. Saad, C. Yin, and M. Debbah, "Artificial neural networks-based machine learning for wireless networks: A tutorial,' IEEE Commun. Surveys Tuts., vol. 21, no. 4, pp. 3039-3071, 4th Quart. 2019.

[27] S. J. Pan, J. T. Kwok, Q. Yang, and J. J. Pan, "Adaptive localization in a dynamic WiFi environment through multi-view learning," in Proc. 22nd Nat. Conf. Artif. Intell., 2007, pp. 1108-1113.

[28] V. W. Zheng, S. J. Pan, Q. Yang, and J. J. Pan, "Transferring multidevice localization models using latent multi-task learning," in Proc. $A A A I$, vol. 8, 2008, pp. 1427-1432.

29] S. J. Pan, D. Shen, Q. Yang, and J. T. Kwok, "Transferring localization models across space," in Proc. 23rd Nat. Conf. Artif. Intell., 2008, pp. 1383-1388.

30] J. Pan, "Feature-based transfer learning with real-world applications," Ph.D. dissertation, Dept. Comput. Sci. Eng., Hong Kong Univ. Sci. Technol., Hong Kong, 2010.

31] E. Baştuğ, M. Bennis, and M. Debbah, "A transfer learning approach for cache-enabled wireless networks," in Proc. 13th Int. Symp. Model. Optim. Mobile Ad Hoc Wireless Netw. (WiOpt), 2015, pp. 161-166.
[32] A. Galindo-Serrano, L. Giupponi, and G. Auer, "Distributed learning 1080 in multiuser OFDMA femtocell networks," in Proc. IEEE Veh. Technol. 108 Conf. (VTC), 2011, pp. 1-6.

[33] W. Wang, J. Zhang, and Q. Zhang, "Transfer learning based diagnosis 1083 for configuration troubleshooting in self-organizing femtocell networks," 1084 in Proc. IEEE Glob. Telecommun. Conf. (GLOBECOM), 2011, pp. 1-5. 1085

[34] M. Chen, W. Saad, C. Yin, and M. Debbah, "Data correlation-aware 1086 resource management in wireless virtual reality (VR): An echo state 1087 transfer learning approach," IEEE Trans. Commun., vol. 67, no. 6, 1088 pp. 4267-4280, Jun. 2019.

[35] Z. Tang, D. Wang, and Z. Zhang, "Recurrent neural network training 1090 with dark knowledge transfer," in Proc. IEEE Int. Conf. Acoust. Speech 1091 Signal Process. (ICASSP), 2016, pp. 5900-5904.

[36] E. U. T. R. Access, "Physical layer-measurements (3gpp ts 36.2141093 version 9.0. 0 release 9)," Standard ETSI TS 136 214, 2010.

[37] F. Afroz, R. Subramanian, R. Heidary, K. Sandrasegaran, and S. Ahmed, 1095 "SINR, RSRP, RSSI and RSRQ measurements in long term evolution 1096 networks," Int. J. Wireless Mobile Netw., vol. 7, no. 4, pp. 113-123, 1097 2015.

1098

[38] I. Goodfellow, Y. Bengio, A. Courville, and Y. Bengio, Deep Learning, 1099 vol. 1. Cambridge, U.K.: MIT Press, 2016.

[39] J. Snoek, H. Larochelle, and R. P. Adams, "Practical Bayesian 1101 optimization of machine learning algorithms," in Proc. 25th Int. Conf. 1102 Neur. Inf. Process. Sys., 2012, pp. 2951-2959.

[40] J. Bergstra and Y. Bengio, "Random search for hyper-parameter 1104 optimization," J. Mach. Learn. Res., vol. 13, no. 2, pp. 281-305, 2012. 1105

[41] F. Chollet et al., (2015). Keras. [Online]. Available: 1106 https://github.com/fchollet/keras $\quad 1107$

[42] M. Abadi et al., "Tensorflow: A system for large-scale machine learn- 1108 ing," in Proc. 12th USENIX Conf. Oper. Syst. Design Implement., 2016, 1109 pp. 265-283.

[43] T. Cover and P. Hart, "Nearest neighbor pattern classification," IEEE 1111 Trans. Inf. Theory, vol. IT-13, no. 1, pp. 21-27, Jan. 1967.

[44] T. K. Ho, "Random decision forests," in Proc. 3rd Int. Conf. Document 1113 Anal. Recognit., 1995, pp. 278-282.

[45] A. Liaw and M. Wiener, "Classification and regression by randomforest," 1115 R News, vol. 2, no. 3, pp. 18-22, 2002.

[46] L. Breiman, "Random forests," Mach. Learn., vol. 45, no. 1, pp. 5-32, 1117 2001.

[47] J. M. Joyce, "Kullback-Leibler divergence," in International 1119 Encyclopedia of Statistical Science. Heidelberg, Germany: Springer, 1120 2011, pp. 720-722.

1121

[48] A. Krizhevsky, I. Sutskever, and G. E. Hinton, "Imagenet classification 1122 with deep convolutional neural networks," in Proc. 25th Int. Conf. Neur. 1123 Inf. Process. Syst., 2012, pp. 1097-1105.

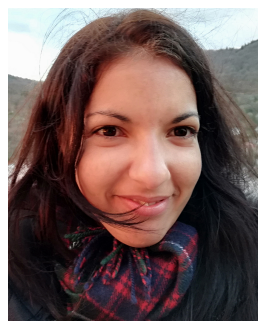

Claudia Parera received the B.S. degree in com- 1125 puter science from the University of Havana, Cuba, 1126 in 2010, and the M.S. degree in advanced computer 1127 science from the University of Bradford, U.K., in 1128 2016. She is currently pursuing the Ph.D. degree 1129 from the Dipartimento di Elettronica, Informazione 1130 e Bioingegneria, Politecnico di Milano. She is 1131 an Early State Researcher in the Anticipatory 1132 Networking Techniques in 5G Networks and Beyond 1133 (ACT5G) Marie Curie project. As part of the current 1134 Ph.D. degree, she is being actively involved in aca- 1135 demic and industrial research with Nokia Bell Labs, Stuttgart. Her research 1136 interests include applying machine and transfer learning techniques to network 1137 analytics, especially in the field of anticipatory networking for 5G networks. 1138

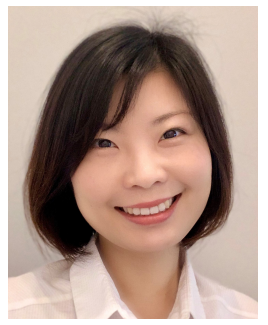

Qi Liao received the M.S. degree in E.E. and 1139 the Dr.-Ing. degree from Heinrich-Hertz-Chair for 1140 Information Theory and Theoretical Information 1141 Technology, Technical University of Berlin in 20101142 and 2016, respectively. From 2010 to 2013, she 1143 was a Research Associate with the Fraunhofer 1144 Institute for Telecommunications, Heinrich Hertz 1145 Institute, Berlin. From 2013 to 2014, she was the 1146 Ph.D. intern with the Department of Statistics and 1147 Learning Research, Bell Labs, Murray Hill, NJ, 1148 USA. Since 2015, she has been a Research Scientist 1149 with the E2E Mobile Network Solutions Lab, Nokia Bell Labs, Stuttgart, 1150 Germany. She holds more than 50 peer reviewed journal articles, conference 1151 papers, and granted or filed patents. Her current research interests include 1152 multiobjective optimizations, optimization for multiagent systems, resource 1153 allocation, stochastic optimization, and machine learning techniques. 


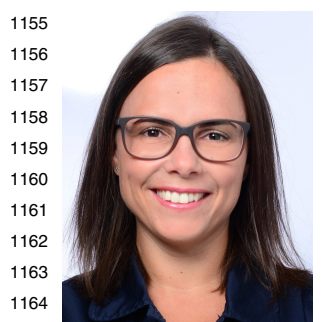

Ilaria Malanchini received the B.S. and M.S. degrees in telecommunications engineering from the Politecnico di Milano, Italy, in 2005 and 2007, respectively, and the $\mathrm{Ph} . \mathrm{D}$. degree in electrical engineering from Drexel University, Philadelphia, and Politecnico di Milano in 2011. She is a Senior Research Engineer with E2E Network \& Service Automation Laboratory and has been with Bell Labs Stuttgart since 2012. She was awarded the MeucciMarconi Award and the Chorafas Foundation Prize for her Master's and Ph.D. thesis, respectively. She 1166 has published more than 25 peer reviewed journal and conference papers and 1167 has more than 10 granted or filed patents. Her research interests focus on 1168 optimization models, mathematical programming, game theory, and machine 1169 learning, with the application of these techniques to wireless network prob1170 lems, such as wireless resource allocation, anticipatory network optimization, 1171 infrastructure and resource sharing, and network slicing.

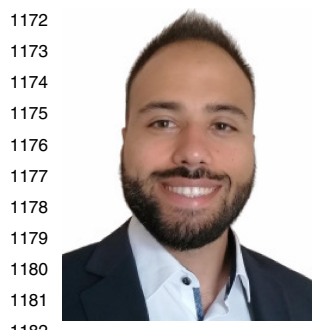

Cristian Tatino received the B.S. and M.S. degrees in telecommunications engineering from the Universitá di Napoli Federico II, Italy, in 2011 and 2013, respectively. He is currently pursuing the Ph.D. degree at the Communications and Transport Systems Division, Department of Science and Technology, Linköping University, Sweden, where he is currently an Early State Researcher within the ACT5G Marie Curie project. From 2013 to 2015 , he worked as a System Engineer on wireless telecommunications networks for railways applica1183 tion. He has been a Visiting Fellow with Nokia Bell Labs, Stuttgart, Germany, 1184 in 2016 and 2018. He focuses his research on the wireless link status 1185 anticipation for millimeter-waves wireless networks. In particular, he studied 1186 the impact of the signal reflections on the coverage probability for non1187 line-of-sight communications, multiconnectivity, and relaying solutions for 1188 communication reliability.

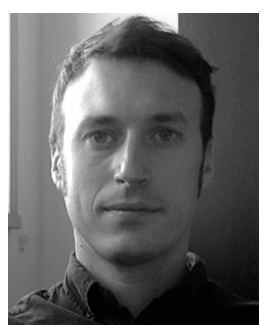

Alessandro E. C. Redondi received the M.S. degree 1189 in computer engineering and the Ph.D. degree in 1190 information engineering from Politecnico di Milano, 1191 Italy, in July 2009 and February 2014, respec- 1192 tively, where he is currently an Assistant Professor 1193 with the Dipartimento di Elettronica, Informazione e 1194 Bioingegneria. From September 2012 to April 2013, 1195 he was a visiting student with the EEE Department, 1196 University College of London. His research activi- 1197 ties are focused on the design and optimization of 1198 IoT systems and on network data analytics.

1199

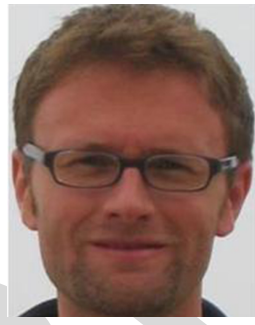

Matteo Cesana received the M.S. degree in 1200 telecommunications engineering and the Ph.D. 1201 degree in information engineering from the 1202 Politecnico di Milano, Italy, in July 2000 and in 1203 September 2004, respectively, where he is a Full 1204 Professor with the Dipartimento di Elettronica, 1205 Informazione e Bioingegneria. From September 1206 2002 to March 2003, he was a Visiting Researcher 1207 with the Computer Science Department, University 1208 of California at Los Angeles (UCLA). His research 1209 activities are in the field of design, optimization, 1210 and performance evaluation of wireless networks with a specific focus on 1211 communication technologies for the Internet of Things and Future Generation 1212 Cellular Networks. He is an Associate Editor of the Ad Hoc Networks 1213 Journal (Elsevier).

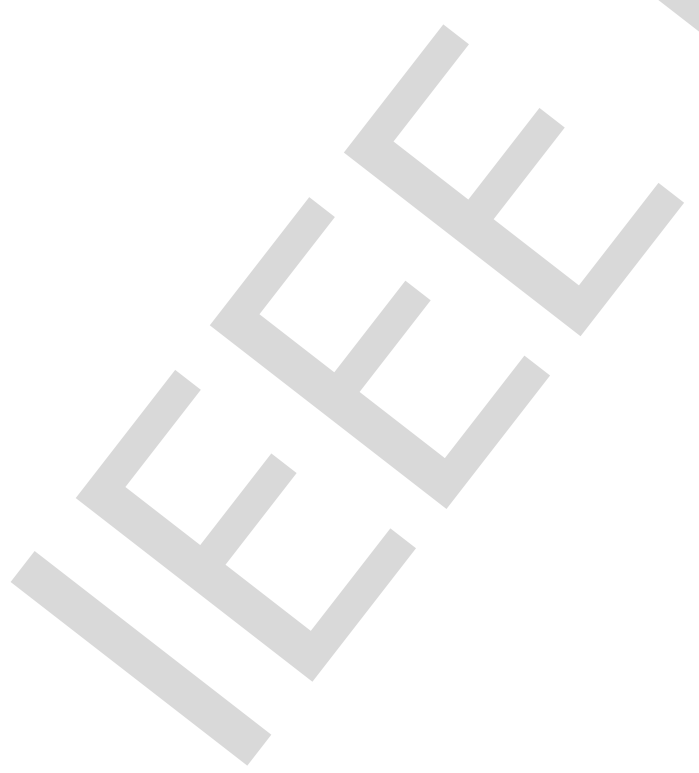

